Journal of

Nutrigenetics

Nutrigenomics
J Nutrigenet Nutrigenomics 2017;10:93-125 Published online: September 7, 2017 DOI: $10.1159 / 000480052$

\title{
11th Congress of the International Society of Nutrigenetics/Nutrigenomics (ISNN)
}

\section{Abstracts}

September 16-19, 2017

Los Angeles, CA
Guest Editors

Jaana Hartiala, Los Angeles, CA

J. Alfredo Martinez, Navarra

Zhaoping Li, Los Angeles, CA

Hooman Allayee, Los Angeles, CA

\section{Contents}

Oral Presentations

Abstracts 01-05

Poster Presentations

Abstracts P5-P66

Author Index 


\section{Journal of \\ Nutrigenetics \\ Nutrigenomics}

\section{Oral Presentations}

\section{1}

\section{Metabolic Responses to Dietary Patterns Differ with Genetic Backgrounds in Mice}

\author{
William Barrington ${ }^{1}$, Carolina Mantilla Rojas², \\ Selene Howe'2, Brian Bennett ${ }^{3}$, Daniel Pomp 4 , \\ David Threadgill ${ }^{2}$
}

1 University of California Los Angeles, Lusis Lab, MedicineCardiology, Los Angeles, CA; ${ }^{2}$ Texas A\&M University, Molecular and Cellular Medicine, College Station, TX;

${ }^{3}$ United States Department of Agriculture, Agricultural Research Service, Davis, CA; ${ }^{4}$ University of North Carolina Chapel Hill, Genetics, Chapel Hill, NC, USA

Dietary patterns have been shown to have profound effects on health when studied at the population level. For example, Japanese and Mediterranean diets are associated with longevity and low rates of chronic disease. The American diet is associated with increased risk of heart disease and certain types of cancer. However, studies evaluating dietary interventions in individuals find significant variations in responses. Much of this variation is likely due to underlying genetic differences among individuals. To determine how diet affects cardiometabolic health in different genetic backgrounds, we compared the health effects of an American diet to a standard mouse diet in each sex of four inbred mouse strains $(\mathrm{A} / \mathrm{J}, \mathrm{C} 57 \mathrm{BL} / 6 \mathrm{~J}, \mathrm{FVB} / \mathrm{NJ}$, and NOD/ShiltJ) selected for their known susceptibilities to cancer, diabetes, and other diseases. Twenty mice per strain were fed each diet for six months while undergoing a range of clinical analyses. All strains had negative health effects when fed the American diet, however the severity of effects varied widely by strain with the most severe effects in C57BL/6J and the mildest effects in A/J. We then investigated the health responses in the same strains fed alternative human-relevant diets including Mediterranean, Japanese, and ketogenic diets. Many diet responses were dependent upon genetic background including: adiposity, glucose tolerance, blood chemistry profiles, liver triglyceride storage, and metabolic rate. The severity and directionality of many of these diet responses differed depending on the genetic background. For example, C57BL/6J maintained good health on a ketogenic diet; FVB/NJ mice became obese and showed signs of cardiometabolic distress on the same diet. Most strains had beneficial effects from the Japanese diet, although abnormal liver effects were identified in $\mathrm{A} / \mathrm{J}$ mice. The Mediterranean diet failed to improve body composition in most strains but improved blood lipids. Mouse strains differed as to which diets were optimal or suboptimal, indicating individuals likely have a specific diet for optimal health based upon their unique genetic makeup. The results emphasize the need to evaluate dietary efficacy at an individual level.

\section{2 \\ Fasting Serum Lipid and Dehydroepiandrosterone Sulfate as Important Metabolites for Detecting Isolated Postchallenge Diabetes: Serum Metabolomics via Ultra-High-Performance LC-MS}

Changhao Sun ${ }^{1}$, Ying $L i^{1}$, Xiaoyan $W^{1}$

${ }^{1}$ Department of Nutrition and Food Hygiene, Public Health College, Harbin Medical University, Harbin, P R China

Background: Isolated postchallenge diabetes (IPD), a subtype of type 2 diabetes mellitus (T2DM) defined as 2-h postprandial plasma glucose $\geq 200 \mathrm{mg} / \mathrm{dL}(\geq 11.1 \mathrm{mmol} / \mathrm{L})$ and fasting plasma glucose $(\mathrm{FPG})<108 \mathrm{mg} / \mathrm{dL}(<6.0 \mathrm{mmol} / \mathrm{L})$, is often overlooked during screening for diabetes on the basis of FPG concentrations. A key challenge is early identification of IPD by the use of fasting serum, which is critical for large-scale diabetes screening.

Methods: We applied a nontargeted metabolomic approach using ultra-high-performance liquid chromatography-quadrupole TOF-mass spectrometry (UPLC-QTOF-MS) to analyze serum samples from 51 patients with IPD, 52 with newly diagnosed T2DM, and 49 healthy individuals. We processed metabolite profiles by multivariate analysis to identify potential metabolites, which were further confirmed by tandem MS (MS/MS). We also used GC-MS and ELISA methods to detect potentially important metabolites. A number of independent samples were selected to validate the identified candidates.

Results: We selected 15 metabolites with a view to distinguishing patients with IPD, whereas 11 were identified with an authentic standard. The selected metabolites included linoleic acid, oleic acid, phospholipids, and dehydroepiandrosterone sulfate (DHEA-S). In IPD samples, significantly higher linoleic and oleic acid $(P<0.001)$ and lower DHEA-S $(P<0.001)$ concentrations were observed, compared with controls. The area under the curve from a combination of linoleic acid, oleic acid, and DHEA-S in the validation study was 0.849 for the IPD group.

\section{KARGER}

(C) 2017 S. Karger AG, Basel 
Conclusions: The current study provides useful information to bridge the gaps in our understanding of the metabolic alterations associated with IPD and might facilitate the characterization of patients with IPD by the use of fasting serum. lower serum cholesterol levels. Large intervention trials are needed to establish the causal link between high milk consumption and cardiometabolic phenotypes before changes in dairy consumption could be recommended for the prevention of cardio-metabolic diseases.
03

\section{Evidence for a Genetic Association between High Milk Consumption and Cardio-Metabolic Traits}

\author{
Vimal Karani ${ }^{1}$, Alana Cavadino ${ }^{2,3}$, Ang Zhou 4 ,Julie A. \\ Lovegrove1, GIANT consortium, Global Lipids Consortium, \\ MAGIC consortium, DIAGRAM consortium, \\ CARDIOGRAM consortium and ICBP consortium, \\ Elina Hyppönen ${ }^{2,4}$ \\ ${ }^{1}$ Hugh Sinclair Unit of Human Nutrition, University of \\ Reading, UK; ${ }^{2}$ Population, Policy and Practice, UCL \\ Institute of Child Health, UK; ${ }^{3}$ Wolfson Institute of \\ Preventive Medicine, Queen Mary University of London, \\ UK; ${ }^{4}$ Centre for Population Health Research, University of \\ South Australia, Adelaide, Australia
}

Studies have shown associations between high milk intake and cardio-metabolic traits. We conducted a genetic study to obtain evidence for the causal relationship between milk consumption and cardio-metabolic traits using the lactase persistence $(L C T-13910 \mathrm{C}>\mathrm{T}$, rs4988235) variant as an instrumental variable in a Mendelian Randomization analysis. We tested the association of $L C T$ genotype with milk consumption (for validation) and with cardio-metabolic traits (for a possible causal association) in three large-scale population studies, with up to 87,790 participants [the 1958 British Birth Cohort $(\mathrm{N}=5,231)$, the Health and Retirement study $(\mathrm{N}=8,520)$ and the UK Biobank $(\mathrm{N}=73,590)]$. The findings from our analyses were confirmed using summary statistics from consortia meta-analyses on intermediate traits [Genetic Investigation of Anthropometric Traits (GIANT, N=327,665), Global Lipids Consortium (N=188,577), the Meta-Analyses of Glucose and Insulin-related traits Consortium (MAGIC, $\mathrm{N}=22,293$ ) and International consortium for Blood Pressure $(\mathrm{N}=146,581)$ and extended to cover disease end-points [DIAGRAM $(34,840$ cases and 114,981 controls $)$ and CARDIOGRAM (22,233 cases and 64,762 controls)]. Carriers of the ' $\mathrm{T}$ ' allele of the $L C T$ variant were more likely to consume milk $\left(\mathrm{P}<3.0 \times 10^{-4}\right)$. In meta-analysis including all three studies, under a recessive model, ' $\mathrm{T}$ ' allele was associated with higher body mass index (BMI), lower low-density lipoprotein (LDL-C) and total (TC) cholesterols. The association of the $L C T$ variant with LDL-C $\left(\mathrm{P}=7.03 \times 10^{-6}\right)$ and TC $\left(\mathrm{P}=1.24 \times 10^{-7}\right)$ was confirmed in the Global Lipids consortium meta-analysis, and the association with $\mathrm{BMI}$ in the GIANT consortium meta-analysis ( $\mathrm{P}=0.001)$. Consortia meta-analyses also suggested an association with lower high-density lipoprotein cholesterol ( $\mathrm{P}=0.001$, Global Lipids), with borderline associations seen for fasting insulin $(\mathrm{P}=0.01$, MAGIC) and HOMA-IR $(\mathrm{P}=0.03$, MAGIC). In consortia meta-analyses, the ' $\mathrm{T}$ ' allele of the $L C T$ variant was associated with a lower risk of coronary artery disease (OR 0.95 , 95\% CI: 0.91-0.99) but not with type 2 diabetes (OR 1.02, 95\% CI: $0.99-1.05)$. In summary, our large-scale study provides genetic evidence for the association of milk consumption with higher BMI but

\section{4 \\ Effects of Prebiotic Fiber Xylo- Oligosaccharides in Adenine Induced Nephropathy in Mice}

\author{
Jieping Yang ${ }^{1}$, Oing $L^{1}{ }^{1}$, Susanne M. Henning ${ }^{1}$, Zhong Jin ${ }^{2}$, \\ Mark Hsu', Rupo Lee ${ }^{1}$, Jianfeng Long ${ }^{1}$, David Heber ${ }^{1}$, \\ Zhaoping $L i^{1, *}$ \\ ${ }^{1}$ Center for Human Nutrition, David Geffen School of \\ Medicine at UCLA, Los Angeles, CA90095, USA; \\ ${ }^{2}$ Department of Pathology \& Laboratory Medicine, David \\ Geffen School of Medicine at UCLA, Los Angeles, CA \\ 90095, USA
}

Background: There is increasing evidence from epidemiological studies showing that high intakes of dietary fiber are associated with decreased risk of chronic kidney disease (CKD), and that patients with CKD have an altered gut microbiota.

Objective: This study evaluated the effect of prebiotic fiber Xylo-oligosaccharides (XOS) supplementation in kidney function and gut microbiome of mice with adenine-induced CKD.

Methods: Male 7-week old C57BL/6 mice were fed with AIN93M control diet or control diet containing $0.2 \%$ adenine $(\mathrm{W} / \mathrm{W})$ for 3 weeks to induced CKD. Four mice from control and adenine group were sacrificed after 3 weeks. Control and CKD mice were then placed on control diet, $3 \%$ or $10 \% \mathrm{XOS}(\mathrm{W} / \mathrm{W})$ for additional 3 weeks ( $n=8 /$ group). Mice were sacrificed after 3 weeks, blood urea nitrogen (BUN), creatinine, kidney pathology, gene expression for fibrosis and inflammatory markers were measured. Cecum content was used for microbiota and short chain fatty acids (SCFAs) analysis.

Results: CKD mice consuming the control diet exhibited increased BUN, creatinine, interstitial fibrosis, inflammation and tubular damages. Supplementation with XOS for 3 weeks significantly attenuated the damages and preserved renal function. 16S rRNA gene sequencing of cecum microbiota revealed that alpha diversity was comparable between CKD mice and control mice, and XOS induced dose dependent decreases of alpha diversity in both $\mathrm{CKD}$ and control mice. Beta-diversity analysis (unweighted Unifrac) showed that samples clustered according to experimental groups. Linear discriminant analysis identified 9 bacterial genera enriched in CKD mice; 6 of these including Dorea, Clostridium, Lactobacillus, Erysipelotrichaceae, Staphylococcus and Allobaculum were significantly reduced with XOS supplementation. XOS increased cecal SCFAs production. Furthermore, cecal SCFAs were negatively correlated with blood levels of BUN.

Conclusion: Our results showed that XOS supplementation improved kidney function in mice with adenine-induced CKD, which was associated with profound changes in gut microbiome composition and the production SCFAs. 


\section{5}

\section{Omics Applications for Obesity Management}

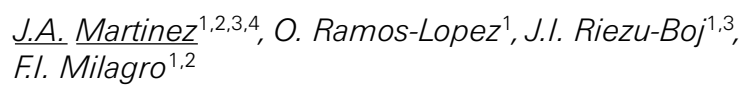

${ }^{1}$ Centre for Nutrition Research/Department of Nutrition,

Food Sciences and Physiology, University of Navarra,

Pamplona, Spain; ${ }^{2}$ CIBERobn, Centro de Investigación

Biomédica en Red de la Fisiopatología de la Obesidad y

Nutrición, Carlos III Health Institute, Madrid, Spain;

${ }^{3}$ Navarra Institute for Health Research (IdiSNA), Pamplona,

Spain; ${ }^{4}$ Madrid Institute of Advanced Studies (IMDEA

Food), Madrid, Spain

The adverse health effects of obesity and accompanying complications remain a major concern due to the scarcity of relevant progress and suitable tools for overweight prevention and treatment. Precision nutrition is an emerging and innovative therapeutic approach that takes into accounts not only the individual's genetic, epigenetic, metagenomic and metabolomic information, but also age, gender, ethnicity, clinical and family history, as well as pathophysiological conditions and treatments or physical activity patterns. In recent years, scientific advances in "omics" sciences are contributing to a better understanding of the role of genetic variants (Nutrigenetics), epigenetic signatures (Nutriepigenetics), gut microbiota composition (Metagenomics), and the metabolome (Metabolomics) in the development of obesity and accompanying complications, and their potential application for therapeutic purposes. For example, interactions between polymorphisms and diet are implicated in disease risks, and also in differential responses to diets restricted in energy or modified in the macronutrient distribution. In this context, studies conducted in several populations have investigated the effects of various SNPs on weight loss, weight regain and reductions in blood lipid levels and insulin resistance. These investigations include SNPs in genes involved in food intake regulation, lipid metabolism, insulin signaling, glucose homeostasis, inflammatory response, and circadian cycle. In addition, experimental studies have investigated the epigenetic mechanisms underlying the beneficial effects of some nutrients and bioactive food components. Based on these evidences, it has been proposed that the introduction of these dietary compounds into an "epigenetic diet" could serve as an effective strategy to reduce the incidence of obesity and associated comorbidities. Also, it has been reported that some epigenetic signatures may be used as biomarkers to follow-up the effect of nutritional treatments on weight loss and improvements in metabolic profiles. Meanwhile, the use of sequencing technologies and metagenomic studies are contributing to the characterization of gut microbiota and the subsequent impact on health. Of particular interest are the effects of dietary carbohydrates and polyphenols on attenuating microbial dysbiosis in the obese through the promotion of "healthy" intestinal bacterial communities for the control of body weight and other metabolic abnormalities related to excessive adiposity. On the other hand, metabolomics focuses on the analysis of changes in the concentration of metabolites in biological samples in order to assess the compliance/adherence to dietary regimens, as well as the identification of new biomarkers related to food intake or disease progression. In this sense, metabolomic analyses are allowing to metabolically categorize individuals depending on dietary intake or nutritional responses. Overall, this sci- entific knowledge based on "nutri-omics" technologies is enabling the design of personalized strategies for obesity management in the era of precision nutrition.

\section{References:}

1 Ramos-Lopez O, Milagro FI, Allayee H, et al: Guide for Current Nutrigenetic, Nutrigenomic, and Nutriepigenetic Approaches for Precision Nutrition Involving the Prevention and Management of Chronic Diseases Associated with Obesity. J Nutrigenet Nutrigenomics 2017;10:4362 .

2 Kohlmeier M, De Caterina R, Ferguson LR, et al: Guide and Position of the International Society of Nutrigenetics/Nutrigenomics on Personalized Nutrition: Part 2 - Ethics, Challenges and Endeavors of Precision Nutrition. J Nutrigenet Nutrigenomics 2016;9:28-46.

3 Ferguson LR, De Caterina R, Görman U, et al: Guide and Position of the International Society of Nutrigenetics/Nutrigenomics on Personalised Nutrition: Part 1 - Fields of Precision Nutrition. J Nutrigenet Nutrigenomics 2016;9:12-27.

\section{Poster Presentations}

\section{P5 \\ Opportunities for Training Nutritional Professionals in Nutritional Genomics: What's Out There?}

\author{
Jorja Collins, *, , , Melissa Adamski*,1, Cliona Twohig ${ }^{1}$, \\ Chiara Murgia ${ }^{1}$ \\ ${ }^{1}$ Department of Nutrition, Dietetics and Food, Monash \\ University, Melbourne, Australia; ${ }^{2}$ Dietetics Department, \\ Eastern Health, Victoria, Australia \\ ${ }^{*}$ These authors contributed equally to this work.
}

Objective: Nutrition intervention tailored to an individual's genetic profile holds promise to improve health outcomes and health expenditure. Nutrition professionals require reputable post graduate training and professional development opportunities to ensure the profession is appropriately placed to deliver healthcare based on a genetic predisposition model. This research aimed to identify and profile training courses available to dietitians and nutritionists in the area of nutritional genomics.

Method: An online search using Google and a snowball search, were conducted using terms related to education and nutritional genomics. Online or face-to-face courses in any country related to, or containing content, on nutritional genomics were included. One-off courses and those no longer accessible were excluded. Key characteristics of courses including cost, duration, content, providers, affiliations and target audience were identified from webpages, with data reported descriptively.

Results: 37 courses varying in duration, content and cost were identified: four post-graduate university degrees, five university course units, four recurring face-to-face workshops, 15 online short courses, eight pre-recorded presentations and one service offering 
regular live webinars. Affiliations with food and pharmaceutical industry (e.g. genetic testing companies), professional organisations and research/education institutes were observed.

Conclusions and Implications: The training courses predominantly delivered online enable nutrition professionals worldwide to upskill in nutritional genomics and personalised nutrition. As courses do not appear equivalent, those seeking training should scrutinise and compare cost, duration, mode, content, and affiliations of course providers to ensure learning needs are met.

P6

\section{Potential Role of Abscisic Acid (ABA) in Protecting Beta Cell Function Under Glucolipotoxic Conditions}

\author{
Fadi Alkayal', Hossein Arefanian', Eman Al Shawaf', \\ Sulaiman Marafie ${ }^{1}$, Ali Tiss ${ }^{1}$ \\ ${ }^{1}$ Research Division, Dasman Diabetes Institute, Kuwait
}

Background: In type 2 diabetes, hyperglycemia in combination with high levels of free fatty acids, a condition known as glucolipotoxicity, affects the mass viability and function of beta cells. The phytohormone abscisic acid (ABA) reportedly induces insulin secretion and might help prevent insulin resistance. In plants, $\mathrm{ABA}$ prevents cell apoptosis and DNA degradation.

Aims: Here, we hypothesized that ABA might have similar protective effects in mammalian beta cells under glucolipotoxic conditions. Thus, we have investigated the effects of ABA on the viability and function of mammalian beta cells treated with high levels of glucose and palmitic acid (PA).

Methods: A rat beta cell line (INS-1) was exposed for $14 \mathrm{~d}$ to 16 $\mathrm{mM}$ glucose with or without $400 \mu \mathrm{M}$ PA in the presence or absence of $10 \mathrm{nM}$ ABA. Label-free proteomic quantification approaches were used to investigate the abundance and differential expression of proteins under these treatment conditions. Proteomics results were validated using RT-PCR and western blotting. Pathway analysis was carried out using Ingenuity Pathways Analysis (IPA) software. Cell viability under each treatment condition was also assessed.

Results: The abundance of key proteins involved in insulin signaling was modulated by ABA in the presence of high levels of glucose and PA. The expression of the transcription factors PDX1, RICTOR, and MAP4K4 was increased under treatment with ABA, while the expression of pathways downstream of ANGPT2 and of pirinixic acid was inhibited under these conditions. Interestingly, canonical signalling pathways such as those involving EIF2, PKA, and the NRF2-mediated Oxidative Stress Response were also clearly modulated by ABA.

Conclusion: Specific pathways and proteins modulated by ABA that were identified in the present study will provide insight into the potential beneficial effects of ABA in protecting beta cells against glucolipotoxic conditions and might promote the use of ABA in the prevention and management of diabetes.

\section{P7}

\section{The FTO Gene's Relationship to Obesity and Type 2 Diabetes}

\author{
Amylee Amos ${ }^{1}$ \\ ${ }^{1}$ Amos Institute, University of Southern California, CA, \\ USA
}

FTO is the obesity and fat mass associated gene in humans and is colloquially referred to as the "Fat Gene". Located on chromosome sixteen, FTO contains a series of single nucleotide polymorphisms (SNPs) that have been studied to determine their relationship to adiposity and type two diabetes. Specifically SNP rs9939609, located within the first intron of FTO, has been studied extensively. Research shows that genotype variants of rs9939609 are correlated with increased BMI, increased waist circumference, altered hunger and satiety related hormones, altered eating behaviors, and greater risk for type two diabetes. While the exact mechanism of this SNP is still unknown, research shows that A allele homozygotes are consistently at higher risk for greater adiposity than are heterozygotes or $\mathrm{T} / \mathrm{T}$ wild types. Many studies have proven that this relationship is independent of glucose levels, hemoglobin A1c, lipid levels, or blood pressure. Additionally, heterozygotes are at increased risk for type two diabetes than are T/T wild types. Much research has been performed to explore potential mediators to these relationships and investigate the mechanism behind the increased disease risk. This literature review analyzed the research of ten studies and the relationship of FTO and obesity and type two diabetes.

\section{P8 \\ Omega-3 Index Predicts Cognitive Impairment Score in an MTHFR-C677T Dependent Manner}

Emma L. Beckett', Charlotte Martin 1 , Katrina King ${ }^{1}$ Suzanne Niblett', Jessica Ferguson', Kylie Abbott', Mark Lucock', Manohar Garg', Martin Veysey ${ }^{1,2}$

${ }^{1}$ The University of Newcastle, Australia; ${ }^{2}$ Hull York Medical School, UK

Background: $\mathrm{B}$ vitamins, plasma homocysteine and omega-3 polyunsaturated fatty acids (Omega-3PUFA) have independently been found to modulate risk for cognitive decline, however results have been mixed. MTHFR-C677T polymorphisms, which alter B vitamin related one carbon metabolism, have also been independently linked to risk for cognitive impairment. Therefore, we investigated the interactions between omega-3 PUFA and MTHFR-C677T genotype in predicting risk for cognitive impairment.

Methods: This study is a secondary analysis of the Retirement Health and Lifestyle Study cohort (an elderly cohort from NSW, Australia). Participants completed the Mini-Mental State Examination (MMSE), and gave blood for assessment of serum folate, $\mathrm{B}_{12}$, homocysteine, omega-3 index (O3I) and MTHFR-C677T genotype (RFLP-PCR). Age, sex, education, income and smoking history were also recorded. Complete data sets were available for 555 individuals (55.8\% female).

J Nutrigenet Nutrigenomics 2017;10:93-125 
Results: $12.1 \%$ of participants had MMSE scores indicative of cognitive impairment. $44.3 \%$ were homozygous for the MTHFRC677T ancestral allele (CC). No parameters varied by allelic carriage, except plasma homocysteine, with higher levels in the subgroup carrying the $\mathrm{T}$ allele.

When all subjects were analysed together (adjusted for serum folate and $\mathrm{B}_{12}$, sex, socioeconomic variables and smoking history) O3I was a significant predictor of a normal MMSE result $(\beta=0.18$, $\left.\mathrm{p}=0.02, \mathrm{R}^{2}=0.12, \mathrm{p}_{\text {model }}=0.0006\right)$. Odds ratio analysis revealed an increased likelihood of a normal MMSE result with each unit rise in O3I [1.19 (1.03-1.38)].

When analysis was stratified by MTHFR-C677T polymorphic allele carriage (CC vs CT/TT), results were only significant in those carrying the $\mathrm{T}$ variant. There was a significant interaction between MTHFR-C677T allelic carriage and O3I in predicting a normal MMSE score $\left(p_{\text {interaction }}=0.02\right)$. A significant interaction was also detected for O3I and serum folate $\left(\mathrm{p}_{\text {interaction }}=0.04\right)$. There was no interaction between O3I and, sex $\left(p_{\text {interaction }}=0.9\right)$, age $\left(p_{\text {interaction }}=0.2\right)$ or serum $B_{12}\left(p_{\text {interaction }}=0.7\right)$. Results were similar when adjusted for plasma homocysteine.

Conclusions: O3I predicts cognitive impairment only in those possessing the MTHFR-C677T polymorphic variant. This variant reduces MTHFR enzyme activity, and may impair B vitamin related one carbon metabolism. Omega-3PUFA may therefore only be beneficial in preventing cognitive impairment in those who have impaired $B$ vitamin metabolism or low folate status.

\section{P9 \\ Rs7903146 and Its Association with Type 2 Diabetes Mellitus}

Dina Ben-Nissan ${ }^{1}$

${ }^{1}$ USC Leonard Davis School of Gerontology, CA, USA

TCF7L2 is among the highly replicated genes presenting a strong association with type 2 diabetes mellitus (T2DM). The single nucleotide polymorphism (SNP) rs7903146 (C/T) on the intron 4 of the TCF7L2 gene has been studied extensively for its link with T2DM risk. In this literature review, ten studies were evaluated in order to understand the relationship between rs7903146 and T2DM. Many studies suggested that the T-allele is indeed related to increased diabetes risk, where people with the homozygous TT allele genotype combination have highest genetic risk for developing this chronic disease. Some of the studies attribute the diabetes prevalence to less incretin secretions, while others attribute it to the beta-cell functionality or decreased insulin secretion. Nonetheless, the true mechanism remains uncertain. This SNP was assessed across varying populations, and was found to have consistent results cross-culturally. Additionally, it was noted that it is one of the strongest SNP's in regard to T2DM risk. As expressed by many of the studies, the Tallele resulted in a disadvantageous trait for those individuals.

\section{P10}

\section{Chemical Stress Induces Up-Regulation of Nurr1: Role of Antioxidants}

\author{
Laura Bordoni ${ }^{1}$, Donatella Fedeli ${ }^{2}$, Cinzia Nasuti ${ }^{2}$, \\ $\overline{\text { Dennis Fiorini', Luana Quassinti' }}{ }^{2}$ Massimo Bramucci², \\ Rosita Gabbianelli ${ }^{1}$ \\ ${ }^{1}$ School of Advanced Studied, ${ }^{2}$ School of Pharmacy and \\ ${ }^{3}$ School of Science and Technology, University of \\ Camerino, Italy
}

Objectives: The nuclear receptor NR4A2 (Nurr1) has been demonstrated to be able to modulate cellular homeostasis, energy metabolism, inflammatory response and dopaminergic system. Nurr1 is induced by stress and injury in brain, and it is necessary for maintenance of midbrain dopaminergic neurons because it regulates the dopaminergic synthesis. Down-regulation of Nurr1 has been observed together with a reduced expression of tyrosine hydroxylase (TH) and an increased level of $\alpha$-synuclein in Parkinson's disease. Finally, a key role in the protection against oxidative and inflammatory stress associated to neurodegeneration has been associated to Nurr1.

The aim of this study was to determine the role of antioxidants in modulating the up-regulation of Nurr1 due to chemical stressor residues in food.

Methods: PC12 dopaminergic cell line has been incubated with the lipophilic chemical stressor permethrin (Perm) in corn oil, for $72 \mathrm{~h}$ with various antioxidants or antioxidants vehicles, like glutathione (GSH), tocotrienols, Extra Virgin Olive oil (EVO) and Electrolyzed Reduced Water (ERW). qPCR of Nurr1 was used to screen the protective effect of the analyzed antioxidants. Corn oil and EVO were analyzed as vehicle for their impact on Nurr1, Nrf2 and NFkB gene expression. Western Blotting analysis of Nurrl and TH was performed as confirm where data showed interesting evidences. Trypan blue was used to count cells and determine cell viability.

Results: Cell viability, reduced in dopaminergic cell line exposed to Perm as chemical stressor, resulted to be improved only by EVO due to its redox and anti-inflammatory capacity, in agreement with its ability to promote Nrf2 and to inhibit NF-kB gene expression; moreover, the two oils, EVO and corn oil, modulated Nurr1 and TH similarly. GSH, tocotrienols and ERW could reduce the up-regulation of Nurr1 due to chemical stress, although ERW was more efficient.

Conclusion: EVO inhibits NF-kB and protects PC12 improving cell viability reduced by Perm. ERW was able to counterbalance the up-regulation of Nurr1 and TH, thus they could be helpful as a dietary tool in the prevention of stressor-induced-dopaminergic neurodegeneration. 


\section{P11}

\section{Vitamin E intake and Pro12Ala Polymorphism of PPARG Gene Interact with Adiponectin Levels}

\author{
Wendy Campos-Pérez', Livier Torres-Castillo", \\ Mariana Pérez-Robles ${ }^{1}$, Erika Martínez-López \\ ${ }^{1}$ Medical Molecular Biology Service, "Fray Antonio \\ Alcalde" Civil Hospital of Guadalajara, Department of \\ Molecular Biology and Genomics, University Center of \\ Health Sciences, University of Guadalajara, Guadalajara, \\ Jalisco, México
}

Background/Aim: The importance of dietary micronutrients intake relies on their many biological functions and high impact on health; unfortunately, micronutrients intake, like vitamin $\mathrm{E}$, is deficient in many populations including countries of southern Asia, United States and México. One of the beneficial effects associated with vitamin $\mathrm{E}$ intake is the enhancing of $P P A R \gamma$ activity and its consequently upregulation of adiponectin expression, nonetheless, there is no reported data about the role of the vitamin E intake on adiponectin levels between PPARG genotypes. The aim of this study was to analyze the adiponectin levels in subjects with the Pro12Ala polymorphism of PPARG according to vitamin E intake.

Methods: A total of 283 subjects were enrolled. Habitual dietary intake was estimated by a validated three-day food consumption record. Total vitamin E intake, either from diet or supplements, was analyzed using the Nutritionist Pro $^{\mathrm{TM}}$ Diet Analysis software. Genomic DNA was extracted from peripheral leukocytes. The Pro12Ala polymorphism (rs1801282) of PPARG was determined by allelic discrimination. Analysis of covariance with Bonferroni posthoc multiple comparison tests were used to analyzed the interaction between genotypes and vitamin $\mathrm{E}$ intake with adiponectin levels.

Results: The $68.9 \%$ were woman, the mean age and BMI of studied population was $37.6 \pm 11.4$ years and $27.4 \pm 7.2 \mathrm{~kg} / \mathrm{m}^{2}$, respectively. Vitamin $\mathrm{E}$ intake was deficient in all subjects with a mean of $1.50 \pm 1.78 \mathrm{mg} /$ day. When serum adiponectin levels were analyzed based on vitamin $\mathrm{E}$ intake among genotypes, it was observed that subjects with higher vitamin E intake and Pro12Ala/Ala12Ala genotype, had statistically significant higher levels of serum adiponectin than subjects with the Pro12Pro genotype (4.4 [3.2-5.7] versus $2.7[2.0-3.5] \mu \mathrm{g} / \mathrm{ml} ; \mathrm{p}=0.024)$. Moreover, subjects with Pro12Pro genotype had higher waist circumference than subjects with Pro12Ala/Ala12Ala genotype $(90.4 \pm 15.0$ versus $87.8 \pm 17.4 \mathrm{~cm}$; $\mathrm{p}=0.027$ ).

Conclusions: Our results suggest that may be necessary to promote a greater consumption of vitamin $\mathrm{E}$ to achieve the intake recommendations that are at least $10 \mathrm{mg}$ /day, especially in subjects with Pro12Pro genotype because they, with lower vitamin E consumption, might be at higher risk to develop metabolic complications.

\section{P12 \\ Blackberry (rubus spp) and Grumixama (eugenia brasiliensis lam) Ellagitannins and Anthocyanins-Enriched Extracts Effects on Growth and mRNA and miRNA Expression in MDA-MB-231 Human Breast Cancer Cells}

\author{
Gabriela Rezende Costa ${ }^{1,2, *}$, Tatiana N. Pedrosa ${ }^{3}$, \\ Renata C. Albuquerque ${ }^{3}$, Silvia Stuchi Maria-Engler ${ }^{3}$, \\ Franco Maria Lajolo ${ }^{1,2}$, Neuza Maria Aymoto Hassimotto ${ }^{1,2}$, \\ Thomas Prates Ong ${ }^{1,2}$ \\ ${ }^{1}$ Food Research Center (FoRC); ${ }^{2}$ Department of Food and \\ Experimental Nutrition, and ${ }^{3}$ Department of Clinical and \\ Toxicological Analyses, Faculty of Pharmaceutical \\ Sciences, University of São Paulo, Brazil \\ email: *costa.gr@usp.br
}

Introduction: Breast cancer is the most prevalent cancer in women. Anthocyanins and ellagitannins found in berries represent promising breast carcinogenesis inhibitory agents. Grumixama (Eugenia brasiliensis Lam), a cherry species from Brazil, and blackberry (Rubus spp) are important sources of anthocyanins and ellagitannins. The aim of this study was to evaluate the effects of grumixama and blackberry ellagitannins and anthocyanins-enriched extracts on growth and RNAs and miRNAs expression in human breast cancer cells.

Methods: MDA-MB-231 (hormone receptors negative) cells were treated with grumixama or blackberry ellagitannins or anthocyanins-enriched extracts. Cell growth analysis (cell cycle, apoptosis and proliferation) was performed. Breast cancer- associated RNAs (84 genes) and miRNAs (84 genes) expression was further evaluated by real time PCR.

Results: grumixama anthocyanins-enriched extract induced cell cycle arrest at G0/G1 in MDA-MB-231 cells. Grumixama and blackberry ellagitannins-enriched extracts also induced cell cycle arrest in G0/G1 and increased cells in subG0. Grumixama and blackberry ellagitannins but not anthocyanins-enriched extracts induced apoptosis. All extracts inhibited cell proliferation. 5 genes (ESR2, FOXA1, JUN, $P T G S 2, V E G F A)$ were up-regulated and 10 genes (ADAM23, ATM, BCL2, CDH1, EGF, GLI1, ID1, MKI67, SNAI2 and THBS1) were down-regulated by these extracts. In addition, these extracts increased expression of $m i R-210$ and blackberry ellagitannins-enriched extract reduced the expression of $m i R-19 a / b$.

Conclusion: Collectively these results suggest that blackberry and grumixama anthocyanins and ellagitannins represent promising agents for inhibition of breast cancer development. Their protective actions involved induction of cell death (apoptosis), inhibition of cell proliferation and modulation of the expression of genes and miRNAs related to apoptosis, cell proliferation and differentiation, aggressiveness, invasion and metastasis.

Financing: FAPESP/Food Research Center (Proc.2013/079148); CNPq (Proc. 142275/2013-1) 


\section{P13 \\ Using Targeted Next-Generation Sequencing as a Cost-Efficient Method to Characterize the Genetics of Salt Sensitivity}

\author{
Olivia Dong 1,2, Oscar Suzuki,2, Mildred Pointer ${ }^{3}$, \\ Martin Kohlmeier4, Tim Wiltshire ${ }^{1,2}$ \\ ${ }^{1}$ Division of Pharmacotherapy and Experimental \\ Therapeutics, UNC Eshelman School of Pharmacy, \\ University of North Carolina at Chapel Hill, Chapel Hill, NC, \\ USA; ${ }^{2}$ Center for Pharmacogenomics and Individualized \\ Therapy, University of North Carolina at Chapel Hill, \\ Chapel Hill, NC, USA; ${ }^{3}$ North Carolina Central University, \\ NC, USA; “UNC Nutrition Research Institute, Kannapolis, \\ NC, USA
}

Background: Genetic variation is associated with inter-individual variability in blood pressure in response to dietary sodium intake (salt-sensitivity, SS). Extensive time is required to assess this phenotype in clinical practice; however, identifying key predictive genetic markers may be a faster and less expensive screening approach. A cost-efficient method allowing for extensive and unbiased interrogation of the genome in large sample sizes is needed to identify key haplotypes and single nucleotide polymorphisms (SNPs) to elucidate the genetics associated with SS.

Objectives: Develop a cost-efficient genetic assay that interrogates extensive genomic regions to identify the genetic markers associated with SS.

Methods: A genetic assay was developed to sequence approximately $124 \mathrm{~kb}$ of the genome consisting of 37 genes (all coding exons and promoter regions for 18 genes; SNPs in 19 genes) associated with SS. The evidence linking these genes to SS varies in strength (i.e. highly correlated or inconclusively correlated). The assay utilizes a custom-capture method using molecular inversion probes to generate a library of DNA fragments for next-generation sequencing (NGS) on the Illumina MiSeq platform. The cost of processing one sample includes DNA extraction, library preparation and NGS, and personnel time.

Results: A MiSeq run multiplexing 62 DNA samples from the 1000 Genomes Project was completed using the genetic assay. Average coverage across all genes for the 62 samples was $200 x$ (range: $8 x-663 x$ ). Four genes had an average of less than 20x average coverage. The cost of processing one sample was $\$ 63$ or $\$ 1.70$ per gene. This cost includes DNA extraction (\$11), library preparation and NGS costs (\$35), and personnel time (\$17).

Conclusions: The preliminary genetic sequencing results indicate further optimization of the assay is needed to ensure all genes have a minimum sequencing depth of 20x for proper genotyping and haplotype calling. The results also suggest it is a promising method for interrogating extensive genomic regions for haplotype and SNPs associated with SS, and the economical cost makes sequencing of large cohorts possible.

P14 - WITHDRAWN

\section{P15 \\ Associations between Nutrient Intake, Visceral Fat and ECHDC3 mRNA Expression in Patients with Different Extents of Coronary Lesion}

Mychelle Kytchia Rodrigues Nunes Duarte', Jéssica Nayara Góes de Araújo², Victor Hugo Rezende Duarte2, Jéssica Bastos Pimenteß ${ }^{3}$, Carolinne Thaisa de Oli veira Fernandes ${ }^{3}$, Angélica Luiza de Sales Souza ${ }^{3}$, Thatyane Oliveira Souza ${ }^{3}$, Juliana Marinho de Oliveira4, Ananilia Medeiros Gomes da Silva ${ }^{5}$, Diego Marques da Costa Santos', Marina Sampaio de Menezes Cruz 6 , Ren ata Caroline Costa de Freitas' ${ }^{2}$ Aline Tuane Oliveira da Cunha', Raquel Suzana Matos de Miranda Torrinhas ${ }^{7}$, Severina Carla Vieira Cunha Lima ${ }^{3}$, André Ducati Luchessi ${ }^{2}$, Vivian Nogueira Silbiger ${ }^{2}$

${ }^{1}$ Postgraduate Program of Nutrition, Federal University of Rio Grande do Norte, Natal, Brazil; ${ }^{2}$ Department of Clinical and Toxicological Analysis, Federal University of Rio Grande do Norte, Natal, Brazil; ${ }^{3}$ Faculty of Nutrition, Federal University of Rio Grande do Norte, Brazil; ${ }^{4}$ Hospital Universitário Onofre Lopes, Federal University of Rio Grande do Norte, Natal, Brazil; ${ }^{5}$ Postgraduate Program of Renorbio, Federal University of Rio Grande do Norte, Natal, Brazil; ${ }^{6}$ Postgraduate Program of Science of Health, Federal University of Rio Grande do Norte, Natal, Brazil; ${ }^{7}$ Department of Gastroenterology (LIM-35), Faculty of Medicine, University of São Paulo, São Paulo, Brazil

This study aimed to assess the relationships among nutrient intake, visceral fat and ECHDC3 mRNA expression in blood of patients with differing extents of coronary lesions. Blood samples were collected from male and female participants $(n=41)$ aged 30-74 years old, who underwent coronary catheterization for serum biochemistry and peripheral blood mRNA expression of ECHDC3 assessments. A 24-hour recall for dietary intake calculation, and anthropometric and bioimpedance analysis for visceral fat mass estimation was also completed for each participant. The extent of coronary lesion was classified according to the Friesinger index (FI). Correlations between the variables studied were tested by Spearman analysis. According to FI, patients were classified: without $(\mathrm{n}=8)$, low $(n=6)$, intermediate $(n=15)$, or major $(n=12)$ lesion. We found a positive correlation of lesion extension with carbohydrate $(\mathrm{r}=0.754, \mathrm{p}=0.002)$, thiamin $(\mathrm{r}=0.507, \mathrm{p}=0.001)$, riboflavin $(\mathrm{r}=0.388, \mathrm{p}=0.012)$, folate $(\mathrm{r}=0.599, \mathrm{p}<0.001)$ and magnesium $(\mathrm{r}=0.528, \mathrm{p}=0.003)$ intakes, and with ECHDC3 expression $(r=0.327, p=0.037)$. ECHDC3 expression was 1.93 -fold higher in patients with intermediate than those with low lesions $(p=0.011)$, 1.91 -fold higher in patients with major than those with low lesions $(\mathrm{p}=0.013)$, and showed a positive correlation with FI $(\mathrm{r}=0.327$, $\mathrm{p}=0.037)$ and visceral fat percentage $(\mathrm{r}=0.416, \mathrm{p}=0.009)$. Our data suggested that the consumption of high amounts of carbohydrates and micronutrients (thiamin, riboflavin, folate and magnesium) may be associated with the development of cardiovascular disease. Moreover, the increase of visceral fat and ECHDC3 upregulation may contribute to disease progression. 


\section{P16}

\section{A Vegan Diet May Lead to Substantial Changes in Gene Regulation by Modifying DNA Methylation Patterns}

Valery Filippov', Karen Jaceldo-Sieg/3, Alexey Eroshkin², Vasiliy Loskutov', Charles Wang ${ }^{1}$, Maria Filippova',

Gary E. Fraser ${ }^{3}$, Penelope Duerksen-Hughes ${ }^{1}$

${ }^{1}$ Department of Basic Sciences, Loma Linda University School of Medicine, Loma Linda, CA 92354; ${ }^{2}$ Sanford

Burnham Prebys Medical Discovery Institute, La Jolla, CA 92037: ${ }^{3}$ School of Public Health, Loma Linda University, Loma Linda, CA 92354, USA

Vegan diets, which avoid all animal based foods, have attracted increasing levels of interest and acceptance in recent years. Vegan diets are believed to confer numerous health benefits that include a decreased rate of aging and mortality, thereby increasing longevity. However, the molecular basis underlying these observed effects are not well-understood. To address this gap, we asked whether there were epigenomic differences between vegans and omnivores by interrogating methylation levels across genomic DNA derived from blood cells in vegans, then compared those findings to similar data obtained in omnivores. Primary analysis identified a significant number of differentially methylated $\mathrm{CpG}$ sites, indicating that the presence or absence of animal derived food can indeed cause epigenetic changes that may affect the regulation of gene expression. Moreover, detailed analysis of genes that are differentially methylated also revealed that many are clustered within a few highly enriched groups of transcriptional factors, each of which has the potential to participate in the regulation of multiple other genes. This finding implies that epigenetic regulation is only one layer of gene expression control, and that the number of nutrition-sensitive genes may be much higher than the number of genes with altered methylomes. Another cluster includes genes that regulate several signal transduction cascades. Interestingly, we found that genes involved in the trafficking of two major opposing neurotransmitters, GABA and glutamate, were differentially methylated. Since the DNA analyzed in this study was derived from blood cells, these findings raise the possibility of novel and unknown functions for these two important biologically active molecules outside of the central nervous system. These studies demonstrate for the first time that differences in food consumption in humans are reflected in extensive epigenetic alterations in the DNA methylation of many genes that play important and sometimes key roles in a variety of biological processes that regulate crucial cell life functions.

\section{P17 \\ Perilipin (PLIN) Gene Polymorphism (rs894160) and Anthropometric Characteristics in a Population Group in the Northeast Region of Brazil}

\author{
Naia Ivo Ferreira', Cecília Rios Mateus Lopes', \\ Vanessa Rosa Oliveira1, Letícia Borges Batinga ${ }^{1}$ \\ ${ }^{1}$ Clinic DNA Nutrition, Brazil
}

Background: Polymorphisms in the perilipin (PLIN) gene have been investigated, in different populations, due to their potential relation with obesity, insulin sensitivity and markers of metabolic syndrome. Moreover, these genes are part of a family of proteins that can be found on lipid droplet surfaces in adipocytes and steroidogenic cells, and plays a central role on lipid storage and breakdown.

Objectives: The aim of the study was to investigate the effect of the PLIN (rs894160) polymorphism on anthropometric variables, such as body mass index (BMI), body fat mass and waist circumference in a population group in the Northeast region of Brazil.

Methods: Cross-sectional study, with 43 individuals of both genders (aged 27-64 y), inhabitants of Northeast region of Brazil, selected by convenience. Body Mass Index (BMI), body fat mass, waist circumference and the PLIN polymorphism (rs894160) were determined. Factorial analysis of variance (ANOVA) was used to evaluate the differences between the genotypes.

Results: Among the participants of the sample, the allele A in rs 894160 was carried by $41,9 \%$. This polymorphism was significantly associated with an increase of waist circumference (by WHO criteria of waist circumference higher than $>94 \mathrm{~cm}$ for men and $>80$ $\mathrm{cm}$ for women) $(\mathrm{p}<0.05)$. The presence of the A allele led to $10 \mathrm{~cm}$ increase in waist circumference. In contrast, the other anthropometric measures, such as BMI and body fat mass, did not present significant difference $(\mathrm{p}>0,05)$.

Conclusion: In this sample, the PLIN polymorphism (rs894160) showed significant association with an increase in waist circumference, what seems to be a genetic influencer over growth of waist circumference. The anthropometric variables BMI and fat mass were not related to the genetic variation, so these interactions require further attention. 


\section{P18 \\ Comparative Analysis of Genome-wide Methylation Profiles of Vegans and Omnivores}

\author{
Valery Filippov', Alexey Eroshkin², Vasiliy Loskutov', \\ Charles Wang 1 , Maria Filippova1', Karen Jaceldo-Siegß', \\ Gary E. Fraser ${ }^{3}$, Penelope Duerksen-Hughes ${ }^{1}$ \\ ${ }^{1}$ Department of Basic Sciences, Loma Linda University \\ School of Medicine, Loma Linda, CA 92354; ${ }^{2}$ Sanford \\ Burnham Prebys Medical Discovery Institute, La Jolla, CA \\ 92037; ${ }^{3}$ School of Public Health, Loma Linda University, \\ Loma Linda, CA 92354, USA
}

DNA methylation is one of the major mechanisms through which epigenetic control of gene expression is achieved in developmental and homeostasis processes. To better understand the role of nutrition in regulating gene expression, we performed DNA methylation genome-wide profiling of blood cell DNA samples isolated from 106 individuals, 38 vegans and 68 omnivores, using the Illumina Infinium HumanMethylation450 platform. Comparative analysis using Partek Genomic software revealed that of the differentially methylated CpG sites identified, the majority are located within known genes. Using two separate analysis engines, IPA and DAVID, we identified several significantly enriched gene clusters and pathways that control vital cellular processes. This includes homeobox and forkhead box transcriptional factors that participate in variety of cell functions, as well as several signaling pathways such as Ras and Wnt. These findings indicate that there is a specific and significant response of the methylome, and likely of the transcriptome, to the nutritional environment. We also found that genes coding for proteins that control cellular transport of two major opposing neurotransmitters, GABA and glutamate, were found to be differentially methylated between vegans and omnivores, indicating their possible important role in responding to nutritional changes in contexts other than the central nervous system. Based on these findings, we conclude that nutritional and dietary habits are able to lead to significant alterations in the methylation landscape in humans. Analysis of these alterations indicate the potential of diet and dietary changes to specifically impact the regulation of genes that participate in important and vital cellular processes.

\section{P19}

\section{Fat Mass and Obesity Associated (FTO) Polymorphisms Interact with Plasma Polyunsaturated Omega 3 Fatty Acid to Influence Inflammation}

\section{Tatiane Mieko de Meneses Fujiii', Marina Maintiguer Norde1, Regina Mara Fisberg', Dirce Maria Lobo Marchioni', Marcelo Macedo Rogero ${ }^{1}$ \\ 1 Department of Nutrition, School of Public Health, University of São Paulo, SP, Brazil}

Objective: This study aimed to investigate the interaction between FTO single nucleotide polymorphisms (SNP), rs8050136, rs9930506, rs9939609, and plasma polyunsaturated omega 3 fatty acid (n-3) profile on an inflammatory score in adults of a cross-sectional population-based study conducted in São Paulo, Brazil.

Methods: All adults $(>19 \mathrm{y}$ and $<60 \mathrm{y})$ participants of the Health Survey-São Paulo who matched the inclusion criteria were selected to participate in the study $(\mathrm{n}=225)$. Smoking habits and physical activity information were obtained using structured questionnaires. Plasma fatty acid profile, eleven plasma inflammatory biomarkers concentration and genotyping of FTO SNP rs8050136, rs9930506; rs9939609 were analyzed from blood samples. Anthropometric measurements were performed in standardized methods. Hardy-Weinberg equilibrium was accessed using a Chi-squared test with continuity correction and the association between inflammatory score and genotypes was conducted using multiple linear regression model adjusted for age, body mass index (BMI), smoking status, gender and physical activity level and the same model was used to test for genotype-plasma fatty acid profile interaction.

Results: All SNP analyzed were in Hardy-Weinberg equilibrium. Inflammation score was positively correlated with waist circumference $(p<0.001)$, BMI $(p<0.001)$, age $(p<0.001)$ and plasma triacylglycerol concentration $(\mathrm{p}=0.041)$, and smoking subjects had statistically higher mean inflammation score than non-smokers $(\mathrm{p}=0.045)$. Statistically significant interaction was found between FTO SNP and n-3, so that homozygous for the major alleles for all the FTO SNP presented lower inflammation score when plasma n-3 level was higher $(\beta(95 \% \mathrm{CI})$ for $\mathrm{rs} 8050136 \mathrm{CC}$, rs9930506AA and rs9939609TT are, respectively: $-1.45(-2.28 ;-0,63)$ and $\mathrm{p}$ inter $=0.005$; $-1.06(-1.76 ;-0.36)$ and $\mathrm{p}$ inter $=0.032 ;-1.51(-2.38 ;-0,64)$ and $\mathrm{p}$ inter $=0.006)$.

Conclusion: FTO SNP genotypes are effect modifiers of the relationship between plasma n-3 levels and inflammation score in adults from Sao Paulo city (Brazil). The replication of these results in other populations can confirm the potential of genomic-personalized nutrition for the prevention of low-grade chronic inflammationrelated diseases.

\section{P20}

\section{APOE Single Nucleotide Polymorphisms Frequencies in a Brazilian Population Sample}

Tatiane Mieko de Meneses Fujiii,2, Monica Yamada', André Luiz Barreto ${ }^{1}$, Maria Aderuza Horst ${ }^{3}$,

Ana Carolina Fonseca', Adriano Bonaldi', Michelle Vilhena1, CintiaVilhena', Ricardo Sobhie Diaz ${ }^{1,4}$

${ }^{1}$ Centro de Genomas Laboratory, Sao Paulo, Brazil; ${ }^{2}$ Department of Nutrition, School of Public Health, University of Sao Paulo, Sao Paulo, Brazil; ${ }^{3}$ Nutrition Faculty, Federal University of Goias, Goiania, Brazil ${ }^{4}$ Department of Medicine, Federal University of Sao Paulo, Sao Paulo, Brazil

Objective: Investigate the frequency of apolipoprotein $\mathrm{E}$ $(A P O E)$ single nucleotide polymorphisms (SNP) in a Brazilian population sample, evaluated by a predictive commercial test, named

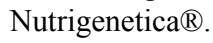


Methods: 295 people mean aged 27 years old participated (196 women and 99 men). We selected patients who did the predictive commercial test since March, 2015 from May, 2017. Briefly, $1 \mathrm{~mL}$ of saliva samples was collected in an Oragene DNA (OG-510) saliva collection device and the allelic discrimination experiment was performed in the Taqman OpenArray ${ }^{\circledR}$ Genotyping system in Centro de Genomas ${ }^{\circledR}$ Laboratory, located in Sao Paulo, Brazil. We genotyped $A P O E$ SNP (rs429358 and rs7412). We calculated de minor allele frequency (MAF) for each SNP and then compared with scientific literature. The reference used was the data from 1000 genomes Project Phase 3, based on CEU population (Utah residents with Northern and Western European Ancestry from the CEPH collection).

Results: All the genetics variants were in Hardy-Weinberg equilibrium. In relation to 1000 genomes Project data, we observed similar MAF frequencies. The $\varepsilon 3 / \varepsilon 3$ genotype was more frequency in this population $(67.1 \%)$, followed by $\varepsilon 3 / \varepsilon 4$ genotype $(18.6 \%) ; \varepsilon 2 / \varepsilon 3$ (11.5\%), $\varepsilon 2 / \varepsilon 4(1.7 \%)$ and $\varepsilon 4 / \varepsilon 4$ (1.0\%). The $\varepsilon 2 / \varepsilon 2$ genotype was not found in this sample.

Conclusion: The presence of $\varepsilon 4$ allele is associated with the increasing risk for late onset Alzheimer's disease (LOAD) and for cardiovascular disease. Alterations in lipid profile can influence both metabolic risks and some studies show the relationship between genotypes and western diet. Genetic variants described in this study may contribute to future research in precision medicine field and may also encourage further intervention studies with the application of personalized nutrition.

\section{P21}

\section{Genetic Architecture of Fatty Liver Due to Dietary Iron Overload in Inbred Strains of Mice}

\author{
Dongyi Lu', Brie Fuqua', Simon Hui', Frode Norheim', \\ Calvin Pan', Sarada Charugundla1, Nam Che1, Brian Parks', \\ Eleazar Eskin', Chris Vulpe ${ }^{2}$, Aldons J. Lusis ${ }^{1}$ \\ ${ }^{1}$ University of California, Los Angeles; ${ }^{2}$ University of \\ Florida, Gainesville, USA
}

Non-alcoholic fatty liver disease (NAFLD) has become one of the most common chronic liver diseases. One-third of NAFLD patients have high liver iron, and it has been hypothesized that iron may contribute to the progression of NAFLD. Previous studies have shown that iron overload can increase hepatic triglyceride and/or cholesterol levels by enhancing lipid synthesis, but the evidence is inconsistent and the mechanism unclear.

We have performed a genome wide association study in 114 strains of hybrid mouse diversity panel (HMDP) male mice to identify novel genetic loci associated with iron metabolism. Mice on the high iron diet developed hepatic iron overload. Although the mice ate less and had lower body fat, hepatic steatosis of various severity was observed histologically, co-localizing with iron loading.

To shed light on the mechanism of how iron overload can lead to steatosis, lipids were extracted from livers of over 700 mice from 114 strains, and triglycerides, total cholesterol, unesterified cholesterol, esterified cholesterol, and phosphatidylcholine were quantified and mapped. Triglycerides, total cholesterol, and phosphatidylcholine mapped most significantly to loci on chromosomes 7, 5, and 16, respectively. Compared to the mice on a high fat, high sucrose diet in a previous HMDP study, mice on the high iron diet had less unesterified cholesterol, less phosphatidylcholine, and more esterified cholesterol, suggesting differences in how these diets affect cholesterol and plasma membrane metabolism. Only a weak correlation was observed in triglyceride and esterified cholesterol levels between the two studies. The previous study mapped liver triglycerides to a different chromosome 7 locus, but this locus was not significant in our mapping. These findings suggest differences in the mechanism of how the two diets lead to steatosis. Using a systems genetics approach, we next aim to integrate these findings with transcriptomic and metallomic data to identify genes and pathways contributing to dietinduced steatosis.

\section{P22 \\ Effect of the Diet on the Transcriptional Expression in Ewes Mammary Gland with Single or Twin Gestation}

\author{
M.A. Gallardo', C. Geoffroy' ${ }^{1}$ L. Arias ${ }^{1}$, S. Hernandez', \\ J.G. Cárcamo \\ ${ }^{1}$ Instituto de Bioquímica y Microbiología, Facultad de \\ Ciencias, Universidad Austral de Chile, PO Box 567, \\ Valdivia, Chile
}

This experiment was carried out to evaluate the effect of the diet on the transcriptional expression levels of some genes related to mamogenesis / angiogenesis processes in the mammary gland in ewes gestating either a single lamb or twins. Eighteen Ile de France ewes, third birth and similar BCS (3.0), 9 gestating a single lamb and 9 gestating twins, were separated in two groups according to the diet consumed: naturalized pasture (NP) or red clover (RC) from day -45 pre-partum until day +70 post-partum. Samples from mammary gland were obtained by biopsy procedure at days -10 pre partum (time 1), +30 (time 2) and +70 post partum (time 3 ). Samples were analyzed by qRT-PCR and data processed using REST statistical software.

Results showed that while ANGPT2 $(\mathrm{P}<0.03)$ and TBAX $(\mathrm{P}<0.01)$ were underexpressed at time 2 in single gestating ewes fed $\mathrm{RC}$, the ewes gestating twins fed RC overexpressed VEGF $(\mathrm{P}<0.01)$, VEGFR1 ( $\mathrm{P}<0.02)$, VEGFR2 $(\mathrm{P}<0.00)$, ANGPT1 $(\mathrm{P}<0.00)$, ANGPT2 $(\mathrm{P}<0.00)$, MK167 $(\mathrm{P}<0.00)$, TBAX1 $(\mathrm{P}<0.01)$, BCL2 $(\mathrm{P}<0.00)$, IGF1 $(\mathrm{P}<0.00)$, IGFBP3 $(\mathrm{P}<0.02)$ and underexpressed TGFB1 $(\mathrm{P}<0.04)$ and TGFB1R1 $(\mathrm{P}<0.02)$ at time 1 . Although at time 2 the ewes only underexpressed CAIV $(\mathrm{P}<0.04)$ and TGFB1 $(\mathrm{P}<0.03)$, overexpressing TGFB1R1 $(\mathrm{P}<0.00)$, at time 3 underexpressed VEGFR1 $(\mathrm{P}<0.04)$, VEGFR2 ( $<<0.04)$, ANGPT1 $(\mathrm{P}<0.04)$, ANGPT2 $(\mathrm{P}<0.03)$, MK167 ( $\mathrm{P}<0.04)$, TBAX1 $(\mathrm{P}<0.02)$, CAIV $(\mathrm{P}<0.05)$, BAX $(\mathrm{P}<0.01)$, BCL2 $(\mathrm{P}<0.04)$, CCND1 $(\mathrm{P}<0.04)$, IGF1 $(\mathrm{P}<0.05)$, LPT $(\mathrm{P}<0.01)$, LPTR $(\mathrm{P}<0.04)$ and IGFB1 $(\mathrm{P}<0.01)$.

It is noticed a transcriptional up-regulation of some genes related to angiogenesis, such as VEGFR1, VEGFR2, ANGPT1, ANGPT2, MK167 and TBAX1 and other genes related to cellular proliferation/ turnover, such as BCL2, IGF1 and IGFB1 at time 1, followed by a downregulation of the same genes at time 3 , suggesting the diet type 
should be a factor to consider in ewes gestating twins but not in single pregnancy.

Funding: InnovaChile 14IDL2-30112. Fondecyt 1150934 and 3160059. FONDAP 15110027.

P23

\section{Identification of miR-612 and miR-1976 as Prospective Biomarkers of Response to Specific Weight-loss Diets}

M. Garcia-Lacarte ${ }^{1}$, F.I. Milagro ${ }^{1,2}$, M.L. Mansego ${ }^{1,2}$, M.A. Zulet 1,2,3, J.A. Martinez $1,2,3,4$

${ }^{1}$ Centre for Nutrition Research/Department of Nutrition, Food Sciences and Physiology, University of Navarra, Pamplona, Spain; ${ }^{2}$ CIBERobn, Centro de Investigación Biomédica en Red de la Fisiopatología de la Obesidad y Nutrición. Carlos III Health Institute, Madrid, Spain; ${ }^{3}$ IdiSNA, Navarra's Health Research Institute, Pamplona, Spain; ${ }^{4}$ Madrid Institute of Advanced Studies (IMDEA

Food), Madrid, Spain

Background: Non-coding RNAs (i.e., miRNAs) play a role in the development of obesity and related comorbidities by regulating gene expression. The aim of this study was to identify candidate miRNA biomarkers throughout -omic approaches in order to predict the success of specific weight-loss dietary treatments.

Materials and Methods: Genomic DNA isolated from white blood cells of a subpopulation from the RESMENA nutritional intervention study was hybridized in Infinium Human Methylation450 BeadChip arrays (31 Low-responders (LR) vs 16 High-responders (RS)), and in Illumina Human HT-12 v4 gene expression BeadChips (14 LR vs $10 \mathrm{HR}$ ). A bioinformatic prediction of putative target sites of selected miRNAs was performed by applying miRBase algorithms.

Results: A total of 134 miRNAs were differentially methylated between HR and LR in the methylation array ( 87 hypomethylated and 47 hypermethylated), whereas 44 miRNAs were differentially expressed between both groups in the expression array (10 downregulated and 34 upregulated). Specifically, miR-1237, miR-1976, miR642, miR-636, miR-612 and miR-193B were simultaneously hypermethylated and downregulated in HR. miR-612 and miR-1976 showed the greatest changes in methylation and expression, respectively; thus, these miRNAs were selected for further evaluation. The bioinformatic prediction revealed that TP53 was a putative target gene of miR-612 and CD40 of miR-1976. Moreover, TP53 was downregulated in the expression array when comparing HR vs LR expression levels adjusted by sex, diet, age and baseline weight $(\mathrm{p}=$ $0.024)$ and CD40 showed a trend $(p=0.069)$. Gene expression levels of TP53 and CD40 were also negatively correlated with the expression of miR612 and miR1976, respectively $(p=0.290 ; R=-0.249$ TP53-miR-612; and $\mathrm{p}=0.023 ; \mathrm{R}=-0.505 \mathrm{CD} 40 \mathrm{miR}-1976$ ).

Conclusion: miR-612 and miR-1976 could be prospective biomarkers of response to specific weight-loss diets and might regulate the expression of TP53 and CD40.

\section{P24}

\section{Determination of Body Fat: A Pilot Study Measuring Body Composition Among College Athletes at a Historically Black University}

\author{
Pratibha Gupta ${ }^{1}$, Aditya Phadke 2 \\ ${ }^{1}$ Central State University, Wilberforce $\mathrm{OH} ;{ }^{2}$ Wright State \\ University, Dayton OH, USA
}

Background: Type 2 diabetes and obesity are major public health priorities because of their high prevalence and incidence. African Americans have the highest prevalence to obesity (29\%) and diabetes (11\%) among racial groups. Obesity and diabetes are strongly associated with other diseases with high mortality rates such as Hypertension, Coronary artery disease, Cancer. The Non-drug treatments included were physical activity and diet lifestyle modification.

Objective: The purpose of this investigation was threefold: 1.Present a profile of body composition assessment using air displacement plethysmography on a population of college athletes at Central State University, 2.Evaluate Body Composition, 3.Determine body fat in College Athletes.

Specific Aims: To determine Body composition, to evaluate and assess specific composition, to Identify risk associated with based on body composition.

Research Methods: We recruited student athletes involved in any sports activities such as volleyball, basketball or any other athletic sport to participate in the study. 51 college athletes male and female, (average age 21) participated in the study. Body Composition measures included \%Fat and \%Fat Free Mass.

Conclusions: A major finding of this study was that a significant number of college athletes had normal and ideal FFMI and FMI. This body composition result could be due to an optimal healthy life style and a particular exercise regimen. Regular assessment of body composition is of importance as changes in body composition have been linked to several health risks. Health studies have shown that a combination of physical activity and a well maintained diet produce the best effect of a decrease in fat mass and a maintenance or increase in muscle mass. Scientists have identified a small number of single nucleotide polymorphisms (SNPs) that respond to diet and exercise. 
P25

\section{Zinc Transporter ZIP13 and Endoplasmic Reticulum (ER) Stress: A Gene-Nutrient Interaction Which May Affect Nervous System Function}

\section{Matthew D. Hart', Morgan D. Strong ${ }^{1}$, Tony Z. Tang ${ }^{1}$, Kaitlyn N. Jerome ${ }^{1}$, Kylin G. Wang1, Brenda J. Smith1, Winyoo Chowanadisai ${ }^{1}$ \\ ${ }^{1}$ Department of Nutritional Sciences, Oklahoma State University, Stillwater, OK, USA}

Implementation of precision nutrition based upon genomic information requires understanding of specific gene-nutrient interactions for distinct health relationships. ZIP13 is an intracellular zinc transport protein which transports zinc into the cytoplasm from internal compartments. Loss of function mutations in ZIP13 cause the spondylocheiro dysplastic form of Ehlers Danlos syndrome, a connective tissue disorder characterized by improper collagen function. However, ZIP13 is widely expressed in nearly all body tissues, including the brain. Accordingly, ZIP13 may play a significant physiological role across multiple body tissues, and mutations or polymorphisms in ZIP13 may be a risk factor for many diseases, including disorders of the nervous system. It has previously been suggested that ZIP13 loss of function induces significant ER stress and the Unfolded Protein Response (UPR). The work presented here supports the hypothesis that ZIP13 is necessary in Neuro-2a neuroblastoma cells for preventing the induction of the ER stress response and promotion of neurite outgrowth, a critical element of neuronal differentiation. Small hairpin RNA mediated ZIP13 knockdown (KD) results in decreased ZIP13 mRNA expression relative to control transfected Neuro-2a cells. ZIP13 KD inhibits neurite extension in Neuro-2a cells differentiated with retinoic acid. ZIP13 KD cells display significant reductions in neurite length, number of neurites per cell, and number of branches per neurite. The observed reduction in neurite outgrowth of ZIP13 KD cells is restored by treatment with sodium tauroursodeoxycholate (TUDCA) and 4-Phenylbutyric acid (4-PBA), compounds which can relieve ER stress. It is possible that genetic variations in ZIP13 leading to reduced function may lead to increased susceptibility to ER stressors from nutritional and environmental sources in the nervous system or other physiological systems.

\section{P26 \\ Interaction Between the Betaine- Homocysteine Methyltransferase (BHMT) Gene Polymorphism and Carbohydrate Intake on Homocysteine Levels in Brazilian Adolescents}

\author{
Shelini Surendran ${ }^{1}$, Carla C. Morais², Julie A. Lovegrove ${ }^{1}$ \\ Cristiane Cominetti², Karani S. Vimal' ${ }^{1}$ Maria A. Horst ${ }^{2}$ \\ ${ }^{1}$ Hugh Sinclair Unit of Human Nutrition, Department of \\ Food and Nutritional Sciences, University of Reading, \\ Reading RG6 6AP, UK; ${ }^{2}$ Nutritional Genomics Research \\ Group, Faculty of Nutrition, Federal University of Goiás \\ (UFG), Goiania, Goiás, Brazil
}

Background: Genetic variants can interact with environmental stimuli such as diet and physical activity, creating variable responses to metabolic traits in several populations. At present, several single nucleotide polymorphisms (SNPs) have been associated with B vitamins, folate and homocysteine status.

Objective: The aim of this study was to investigate the association between MTHFR, MTR, MTRR, CBS, TCN2, COMT, BHMT, FUT2 and NBPF3 gene polymorphisms with vitamins B12, homocysteine and folic acid blood levels and investigate whether dietary factors and physical activity modified the possible associations.

Design: A total of 115 adolescents (10-19 years old), from a public school in Brazil underwent anthropometric, biochemical and genotyping analyses. Dietary intake was estimated with food records and physical activity was based upon self-report. Interaction analyses were performed using linear/logistic regression model. Potential confounders such as age, body mass index, sex were adjusted in the regression model.

Results: None of the associations between the analysed SNPs and vitamin B12, homocysteine and folic acid concentrations were statistically significant after correction for multiple testing. In the interaction analysis, after Bonferroni correction, there was a significant interaction between BHMT SNP (rs689415) and carbohydrate intake $\left(\%\right.$ energy) $\left(\mathrm{P}_{\text {interaction }}=5 \times 10^{-6}\right)$ on homocysteine levels, where the ' $\mathrm{G}$ ' allele carriers had $38.04 \%$ higher circulating homocysteine concentrations than those with 'AA' genotype $(\mathrm{P}=0.04)$ among individuals in the highest tertile of carbohydrate intake (\% energy, 71.56 \pm 12.79 ).

Conclusion: Our findings suggest that the association between the BHMT SNP (rs689415) and circulating homocysteine might be influenced by carbohydrate intake in adolescents. Further understanding of how the BHMT influences circulating homocysteine through dietary interventions is imperative to advance the development of personalised nutrition, to reduce the risk of elevated homocysteine concentrations. The limitation of this study includes a self-report physical activity and no evaluation of betaine and BHMT protein levels. 


\section{P27}

Analysis of Intestinal Microbiota in Patients with Functional Constipation After Synbiotics Treatment

\author{
Lin sheng Huang, Tong yi Shen, Xuebing Yan, \\ Huan long Qin
}

Department of General Surgery, Shanghai Tenth People's Hospital affiliated to Tongji University No.301, Yanchang

Road, Shanghai 200072, China

Objectives: The aim of this study was to investigate whether clinical symptoms and intestinal microbiota is improved after synbiotics treatment.

Methods: Fecal samples from 53 patients diagnosed with chronic functional constipation according to the Rome III criteria were analyzed by $16 \mathrm{~S}-\mathrm{rRNA}$ sequencing. After Synbiotics treatment for one and three month, fecal samples collected from 36 patients and 15 patients respectively. The clinical outcome and intestinal microbiota of patients were then analyzed and 53 healthy community volunteers were employed as controls. To determine the most discriminative species, cross validated logistic ridge regression was performed.

Results: The microbiota composition of constipation group is different from that of treatment group and healthy group. With synbiotics treatment 1 month and 3 month, abundence of EscherichiaShigella is decreased, that of Prevotella_9 and Lactococcus are increased. Compared with constipation and healthy group, genus of Parabacteroides,Erysipelotrichaceae_UCG-003were abundent in the constipation group, whilePrevotella_9,Megamonas, Klebsiella,

Enterobacteriaceae_unclassifiedare abundent in the healthy group.compared microbiota in three groups (constipation group, healthy group as well as treatment group) Prevotella_9 is the characteristic bacteria that decreased in constipation group and increased in treatment group. $(2.12 \%$ vs. $14.46 \%$ vs. $5.65 \%, P=1.13 \mathrm{E}-07, q=$ 3.21E-06, FDR=7.56E-06). after synbiotics treatment,stool frequency is increased and the proportion of constipated complications (abdominal distension, loss of appetite, anxiety rate) is decreased, the proportion of laxative dependent is also decreased.

Conclusions: Synbiotics treatment can promote clinical remission and microbiota improvement in constipation group. Identification of the key bacterial genus is an important approach to reveal therapeutical mechanism of synbiotics treatment, and therefore promotes the development of microbiota-based individualized treatment in patients with functional constipation.

\section{P28}

\section{The Role of Vitamin E on Tight Junction Proteins of Intestinal Barrier}

Yanjun Huang 1,2, Sichun Zhou',2, Caimei He ${ }^{1,2}$, Ting TaO 1,2, Huansheng Yang ${ }^{3,4}$, Yulong Yin ${ }^{3,4}$, Xiaoping Yang 1,2,3

${ }^{1}$ Department of Pharmacy, School of Medicine, Hunan Normal University, Changsha, Hunan, P.R. China 410013; ${ }^{2}$ Key Laboratory of Small Targeted Molecules' Discovery, Hunan Normal University, Changsha, Hunan, P.R. China 410013; ${ }^{3}$ Animal Nutrition and Human Health Laboratory, School of Life Sciences, Hunan Normal University, Changsha, Hunan 410125, China; ${ }^{4}$ Chinese Academy of Sciences, Institute of Subtropical Agriculture, Key Laboratory of Agroecological Processes in Subtropical Region, Changsha 410125, China

Although application of vitamin E has been long time used in both human and animal nutrition worldwide, the detailed mechanisms are largely unknown. Tight junction (TJ) proteins including occludin, claudin-1 and ZO-1 play an important role in intestinal nutrition. We aim to examine the regulatory role of vitamin $\mathrm{E}$ on $\mathrm{TJ}$ proteins. Caco-2 and IPEC-J2 cell lines are treated with vitamin E alone or LPS and the alterations of these TJ protein expressions were measured by either western blot or RT-PCR. The results demonstrate that vitamin $\mathrm{E}$ dramatically increases the expression of $\mathrm{TJ}$ proteins and it relieves activation of IL- 6 and NF- $\mathrm{B}$ after LPS stimulation. The data collected from intestine tissues from vitamin E-treated weaning suckling pigs consistently reveals the similar findings. Altogether, the present study shows a novel role of vitamin E on protecting the intestinal barrier and improving the inflammation via affecting tight junction proteins in intestine. These results build a solid foundation for appropriate utilization of vitamin $\mathrm{E}$ with suitable dose on both human and animal nutrition.

\section{P29}

Genetic Variation in Mitochondrial Complex
I Activity Among Inbred Strains of Mice

Simon T. Hui ${ }^{1}$, Telisha M. Swain ${ }^{2}$, Calvin Pan',

Maria De Luca ${ }^{3}$, Shannon M. Bailey², Aldons J. Lusis ${ }^{1}$

${ }^{1}$ Department of Medicine, Division of Cardiology, David Geffen School of Medicine, University of California at Los Angeles, Los Angeles, CA 90095, USA; ${ }^{2}$ Department of Pathology, University of Alabama at Birmingham, Birmingham, AL 35233, USA; ${ }^{3}$ Department of Nutrition Sciences, University of Alabama at Birmingham, Birmingham, AL 35233, USA

Mitochondrial dysfunction has been shown to link to metabolic diseases, such as obesity, NAFLD and diabetes. To dissect the genetic control of mitochondrial functions and its physiological role, we employed a panel of $>100$ strains of inbred mice, termed Hybrid Mouse Diversity Panel (HMDP), which allows for high resolution mapping. Previously, we have shown that when fed a high fat/high 
sucrose obesogenic diet, HMDP mice exhibited large variations in obesity, insulin resistance and hepatic steatosis which are dependent on their genetic background. We isolated mitochondria from 287 livers from 102 strains of male mice. Complex I activity was measured and normalized to citrate synthase activity. A 10-fold difference was observed between the extreme strains. We found that complex I activity was negatively correlated with obesity traits: body weight, body fat percentage, fat mass response to diet. Parallel to the obesity connection, complex I activity was inversely correlated with food intake and HOMA-IR. These data show that complex I activity is tightly associated with body composition and insulin action. Metabolomic analysis revealed a negative correlation between complex I activity and plasma gamma-aminobutyric acid (GABA). In the liver, complex I activity showed a negative correlation with 4-aminobutyrate aminotransferase (Abat), an enzyme that catalyzes GABA catabolism and is involved in the mitochondrial nucleoside salvage pathway. Our finding is consistent with previous report that human subjects harboring homozygous ABAT missense mutation displayed increased GABA level and reduced mtDNA in the brain. These data suggest that GABA may be a useful plasma biomarker for mitochondrial function. Genome wide association mapping identified two significant loci on chromosomes 8 and 13 . The chromosome 8 peak spans $\sim 1.4 \mathrm{Mb}$ and contains 18 genes whereas the chromosome 13 peaks is $\sim 1.7 \mathrm{Mb}$ wide containing 4 genes. The identification and validation of causal genes in these loci will provide novel insight into the regulation of complex I function by nuclear genes.

\section{P30 \\ Effects of Probiotic Supplementation on Gut Microbiota and Obesity Outcomes in Obese Hispanic Adolescents: A 16-Week, Randomized, Placebo-Controlled Trial}

Roshonda B. Jones', Tanya L. Alderete', Ashley A. Martin', Bree A. Geary', Darryl H. Hwang', Suzanne L. Palmer', Michael I. Goran ${ }^{1}$

${ }^{1}$ Department of Preventive Medicine, University of Southern California, Los Angeles, CA, USA; ${ }^{2}$ Department of Radiology, University of Southern California, Los Angeles, CA, USA

Numerous studies have shown that there are links between obesity, liver fat, and the gut microbiome. However, there are mixed results on whether probiotics could impact the gut microbiome and/or help to decrease liver fat and obesity outcomes. The primary aim of this study was to determine whether a probiotic supplement (VSL $\# 3^{\circledR}$ ) intervention altered gut microbiota and/or gut hormones associated with appetite regulation. The secondary aim of this study was to determine whether VSL $\# 3^{\circledR}$ altered body composition and liver fat and fibrosis. We conducted a double-blind, randomized placebo controlled trial in 19 obese Latino adolescents. Adolescents received three packets per day of either VSL $\# 3^{\circledR}$ or a matched placebo for 16 weeks. Pre- and post-intervention measures included gut bacterial relative abundance, gut appetite regulating hormones, anthropometrics, body composition, liver fat, liver fibrosis and dietary intake. We conducted linear models to determine whether there were any signifi- cant differences in the changes in these outcomes following VSL\#3 ${ }^{\circledR}$ intervention. Models adjusted for sex, change in caloric intake, and body mass index (where appropriate). Compared to placebo, adolescents that received VSL\#3 had significant increases in total adiposity $(\%)(+1.7 \pm 0.6 \mathrm{vs}-1.3 \pm 0.5, p<0.01)$ and trunk adiposity $(\%)(+3.3$ \pm 0.8 vs $-1.8 \pm 0.8, p<0.01)$ with no significant effects on liver fat/ fibrosis, insulin/glucose, gut microbial abundances or gut hormones. We conclude that VSL\#3 supplementation may lead to increased adiposity in obese Latino adolescents with no significant detectable changes in gut microbiota, gut appetite-regulating hormones, liver fat and fibrosis and dietary intake.

P31

Association between Serum Zinc Level and Body Composition: The Korean National Health and Nutrition Examination Survey

\author{
Ha-Na Kim ${ }^{1}$, Sang-Wook Song ${ }^{1}$, Whan-Seok Choi ${ }^{2}$ \\ ${ }^{1}$ Department of Family Medicine, College of Medicine, St. \\ Vincent's Hospital, The Catholic University of Korea, \\ Seoul, Republic of Korea; ${ }^{2}$ Department of Family \\ Medicine, College of Medicine, Seoul St. Mary's Hospital, \\ The Catholic University of Korea, Seoul, Republic of Korea
}

Objective: We examined the associations between serum zinc levels and several body composition factors among Korean adults.

Methods: This study used data from the Korean National Health and Nutrition Examination Survey, a cross-sectional survey of Korean civilians. Data from a total of 1896 subjects were analyzed.

Results: Serum zinc levels in men with elevated waist circumference were higher than in those with normal waist circumference $(152.1 \pm 3.7 \mu \mathrm{g} / \mathrm{dL} v s .137 .8 \pm 2.2 \mu \mathrm{g} / \mathrm{dL}, P<0.001)$ and serum zinc levels increased with increasing tertiles of total body fat percentage $(134.2 \pm 2.8 \mu \mathrm{g} / \mathrm{dL}, 142.0 \pm 2.9 \mu \mathrm{g} / \mathrm{dL}$, and $148.0 \pm 2.7 \mu \mathrm{g} / \mathrm{dL}, P=$ 0.001 ). Among men with a normal waist circumference, the serum zinc levels of participants with the highest total body fat percentage were higher than in those with the lowest or medium total body fat percentage values $(145.4 \mu \mathrm{g} / \mathrm{dL} v s .135 .2 \mu \mathrm{g} / \mathrm{dL}, P=0.029)$. In contrast, in men with an elevated waist circumference, no difference in serum zinc levels according to total body fat percentage was detected. There was no relationship between serum zinc levels and body composition factors in women.

Conclusions: Body zinc status might be associated with the quantity and distribution of body fat in Korean men. Additional gender-specific studies are needed to determine whether the relationship of body zinc status with abdominal obesity and total body fat affects metabolic disorders and cardiovascular diseases.

P32 - WITHDRAWN 
P33

Role of Sex- and Tissue- Specific Differences in Mouse Lipocalin-2 Expression in Regulating Insulin Resistance and NonAlcoholic Fatty Liver Disease Progression

\author{
Karthickeyan Chella Krishnan ${ }^{1}$, Simon Sabir ${ }^{2}$, Calvin Pan ${ }^{1}$, \\ Nam Che ${ }^{1}$, Sarada Charugundla ${ }^{1}$, Hannah Q $i^{1}$ \\ Zhiqiang Zhou', Simon Hui', Frode Norheim', \\ Aldons J. Lusis ${ }^{1}$ \\ 'Department of Medicine/Division of Cardiology, David \\ Geffen School of Medicine, University of California, Los \\ Angeles, CA, USA; ${ }^{2}$ Department of Psychology, University \\ of California, Los Angeles, CA, USA
}

Non-alcoholic fatty liver disease (NAFLD) is an umbrella term encompassing a range of liver abnormalities from simple steatosis (fat accumulation in hepatocytes) to complex non-alcoholic steatohepatitis (NASH), fibrosis and cirrhosis. The prevalence of NAFLD in the United States has increased dramatically over the past few decades. Population studies have strongly associated obesity, diabetes and insulin resistance (IR) to NAFLD. The regulation of secreted cytokines and proteins from adipose tissue, aka adipokines, and liver tissue, aka hepatokines is known to contribute to the pathogenesis of several cardiometabolic diseases including obesity, IR, NAFLD and atherosclerosis via their crosstalk between themselves, adipose and liver, and other tissues such as heart and skeletal muscle. Numerous adipokines and hepatokines have been identified so far; however, specific signalling pathways activated by most of these secreted proteins and/or mechanism(s) by which they communicate with each other (and other tissues) are currently unknown. It is therefore essential to determine the functional consequences of these proteins in regulating normal metabolic homeostasis in order to understand how they are disrupted in patients with obesity, IR and NAFLD. To determine the causal mechanism(s) mediating diet-induced obesity, IR and NAFLD, our lab subjected both sexes of over 100 inbred mice strains to high-fat, high-sucrose (HF/HS) diet for 8 weeks, collecting several metabolic disease associated phenotypes including adipose and liver transcriptomes. Extensive systems genetics analyses of these data suggest that female adipose and/or male liver derived lipocalin-2 (LCN2) may be mechanistically linked to deregulated lipid accumulation in liver, as well as enhanced inflammation in adipose and/or liver. LCN2 is a $25 \mathrm{kDa}$ secretory glycoprotein, first isolated from neutrophils; however, recently it has been shown to be a novel adipokine that is up regulated in adipose tissues of genetically obese animals and humans. LCN2 has also been shown to be a hepatokine associated with liver damage, inflammation and IR. Recently, LCN2 has also been implicated in recruiting neutrophils to the liver in both alcoholic and non-alcoholic steatohepatitis (ASH and NASH), a severe condition of the liver. The goal of our current study is to use systems genetics approaches to investigate the role and mechanism by which LCN2, a secreted protein from adipose and/or liver tissue, in response to diet-induced obesity, regulates IR and NAFLD in a sex-specific manner.

\section{P34}

\section{Dietary Saturated Fat Levels and Protein Source Effects on the Gut Microbiome}

\author{
Jennifer M. Lang1 ${ }^{1}$ Ira Kurtz², Stanley L. Hazen³, Nathalie \\ Bergeron $^{4,5}$ Ronald M. Krauss ${ }^{5}$, A. Jake Lusis $^{1}$ \\ ${ }^{1}$ Division of Cardiology, UCLA Department of Medicine, \\ 650 Charles E Young Dr South, CHS A2-237, Los Angeles, \\ CA 90095, USA; ${ }^{2}$ Division of Nephrology, UCLA \\ Department of Medicine, 650 Charles E Young Dr South, \\ 7-155 Factor, Los Angeles, CA 90095, USA; ${ }^{3}$ Lerner \\ Research Institute, Cleveland Clinic, 9500 Euclid Ave, \\ Cleveland, $\mathrm{OH}$ 44106, USA; ${ }^{4}$ Touro University California, \\ 1310 Club Drive, Vallejo, CA 94952, USA; ${ }^{5}$ Children's \\ Hospital Oakland Research Institute, 5700 Martin Luther \\ King Jr. Way, Oakland, CA 94609, USA
}

The effect of interacting nutrients on disease risk and host physiology is difficult to determine in humans. The Animal and Plant Protein And Cardiovascular Health (APPROACH) study was a randomized controlled human dietary intervention trial aimed at determining whether protein source interacts with saturated fat intake to influence blood lipoproteins and risk for cardiovascular disease. Because the microbiome is known to be involved in nutrient processing and disease risk, the present study evaluated changes of the microbiome in response to these dietary changes. The participants were 110 healthy normolipidemic men and women aged 21-65, with BMI of 20-35. After a $2 \mathrm{wk}$ baseline diet, they were randomized to high $(15 \% \mathrm{E})$ or low $(7 \% \mathrm{E})$ saturated fat groups and randomly received diets ( $4 \mathrm{wks})$ in which the major protein source $(25 \% \mathrm{E})$ was red meat (beef, pork), white meat (chicken, turkey), and non-meat sources (nuts, beans, soy). All participants received all protein source diets, and there was a two-week washout period between diets where participants were on their usual home diet. Taxonomic characterization of fecal microbial communities collected at diet completion was performed by sequencing $16 \mathrm{~S}$ ribosomal RNA gene amplicons. The overall effect of these diets did not significantly alter the microbiome community in PCoA ordination space. Interpersonal differences were greater than dietary influence, and most samples clustered by patient. The significant factors influencing community composition were age, triglycerides, body fat (\%), systolic blood pressure, height, diet order, sex, and alpha diversity. In addition, alpha diversity was positively correlated with age and HDL cholesterol and negatively correlated with triglycerides and systolic blood pressure. When looking more specifically at differential abundances of taxa between diets, there were 7 significantly different genera and 175 different OTUs between high and low saturated fat. There were no significant differences between red and white meat, but 5 OTUs were significantly different for each red meat vs. non-meat and white meat vs. non-meat. Three of those OTUs were consistent between the comparisons. The results demonstrate that inter-individual differences outweigh these specific dietary changes, but there are still taxa that respond to the changing nutrients. 


\section{P35 \\ Xylo-Oligosaccharides Suppressed Visceral Fat Accumulation and Modulated Cecum Microbiome in Mice}

\author{
Jianfeng Long ${ }^{1}$, Jieping Yang ${ }^{1}$, Qing Li', \\ Susanne M. Henning ${ }^{1}$, Mark Hsu', Brenda Chan', \\ Shih Lung Woo ${ }^{1}$, David Heber ${ }^{1}$, Zhaoping Li, * \\ ${ }^{1}$ Center for Human Nutrition, David Geffen School of \\ Medicine at UCLA, Los Angeles, CA 90095, USA
}

Background: Xylo-oligosaccharides (XOS) is a fiber that has been shown to improve the balance of gut microbiota and may improve insulin sensitivity and glycemic control, however, the metabolic regulatory effects of XOS remain unclear.

Objective: This study evaluated the metabolic regulatory effect of prebiotic fiber XOS supplementation in obese mice.

Methods: Male C57BL/6 mice were fed with AIN93M diet for 3 weeks, then were divided into three groups - control diet, $3 \%$-, or $10 \%-\mathrm{XOS}(\mathrm{W} / \mathrm{W})$ for additional 3 weeks ( $\mathrm{n}=8 /$ group). Body composition, liver and blood lipids, and gene expressions related to lipid homeostasis were measured. Cecum content was used for microbiota and short chain fatty acids (SCFAs) analysis.

Result: The body weight, liver weight, liver and blood lipids (total cholesterol and triglycerides), as well as beige adipogenic genes of E-fat did not differ among the three groups. $10 \%$ XOS supplementation significantly reduced the visceral fat, serum leptin, as well as mRNA expression of adipogenic genes PPAR $\gamma$ and $\mathrm{C} / \mathrm{EBP} \alpha$ in epididymal fat (E-fat). In addition, XOS supplementation increased cecum content and decreased alpha diversity of cecal microbiota in a dose-dependent manner. Unweighted $\beta$-diversity analysis showed distinct clusters of microbiota after 3 weeks of XOS treatment. 10\% XOS supplementation favorably increased the ratio of Bacteroidetes to Firmicutes when compared to both control and 3\% XOS supplementation. In addition, XOS (both $3 \%$ and $10 \%$ ) supplementation resulted in significantly increased levels of cecum, but not blood, SCFAs compared with control mice.

Conclusion: In summary, $10 \%$ XOS supplementation reduced visceral fat, and increased ratio of Bacteroidetes to Firmicutes and cecum SCFAs levels. XOS may mediate microbial changes, resulting in increased production of SCFAs, of which might modulate the metabolic environment of the host as shown by decreased mRNA expression of adipogenic genes in adipose tissue. The mechanism of how bacterial changes modulate adipogenesis in visceral adipose should be elucidated.

\section{P36}

\section{Identification and Validation of HIF-1 $\alpha-$ Regulated Genes in 3T3-L1 Adipocytes Treated with Conditioned Medium}

\author{
A. López-Pascual' ${ }^{1}$, S. Lorente-Cebrián ${ }^{1,2}$, J.A. Martínez ${ }^{1,2,3}$, \\ P. González-Muniesa ${ }^{1,2,3}$ \\ ${ }^{1}$ Centre for Nutrition Research/Department of Nutrition \\ and Food Sciences and Physiology, University of Navarra, \\ Pamplona, Spain; ${ }^{2}$ Health Research Institute of Navarra \\ (IdiSNA), Pamplona, Spain; ${ }^{3}$ CIBERobn Physiopathology of \\ Obesity and Nutrition, Centre of Biomedical Research \\ Network, ISCIII, Madrid, Spain
}

Background: Obesity is a multifactorial chronic inflammatory disease which involves different pathways, being adipose tissue hypoxia among them. The aim of the present study was to characterize the effect of conditioned medium from LPS-activated macrophages on the regulation of HIF-1a-related genes in murine adipocytes.

Methods: The identification of HIF-1 $\alpha$-binding sites of 17 obesity-related genes was performed by applying bioinformatics predictions (PROMO web version 3.0.2). For the in vitro analyses, 3T3-L1 murine adipocytes ( 9 days post-differentiation) were incubated for $24 \mathrm{~h}$ in conditioned medium (CM, composed by $75 \%$ complete medium and $25 \%$ medium of RAW 264.7 murine macrophages with $24 \mathrm{~h} 500 \mathrm{ng} / \mathrm{ml}$ LPS). In order to elucidate if these genes were regulated by HIF- 1a, a validation with chromatin immunoprecipitation (ChIP-q-PCR) assay was carried out.

Results: ChIP assay showed that HIF- $1 \alpha$ directly bound to HIFresponsive elements (HRE) in Vegfa and Ucp2 genes, regulating its expression. The conditioned medium increased the binding of HIF-1a at Vegfa $(\mathrm{p}<0.001)$ whereas it suppresses the enrichment at the Ucp2 gene $(p<0.05)$. All these results were statistically significant compared to non-treated cells as a negative control.

Conclusions: Our results suggest that Vegfa and Ucp 2 genes are direct targets of HIF-1a in 3T3-L1 adipocytes treated with conditioned medium from LPS-activated macrophages. The involvement of HIF-1a under pro-inflammatory conditions could help to clarify the role of this transcription factor in obesity and the associated comorbidities. Finally, this study propose a possible application of conditioned medium as a model of macrophage infiltration commonly found in the adipose tissue of obese subjects. 
P37

\section{Altered Eating Disinhibition Genotype Associated with Higher Body Mass Index in Weight Loss Clinic Patients}

\author{
Lori E. Arguello1, Kasuen Mauldin² ${ }^{2}$, Deepika Goya/ \\ ${ }^{1}$ The Hernried Center Weight Loss Clinic, Sacramento, \\ CA; ${ }^{2}$ Department of Nutrition, Food Science, and \\ Packaging, San José State University, San José, CA; \\ ${ }^{3}$ The Valley Foundation School of Nursing, San José State \\ University, San José, CA, USA
}

The purpose of this study was to (a) identify via chart review the type and frequency of six eating behavior genotypes, or the various forms of a gene, in a sample of patients in a medical weight loss clinic and (b) compare results by patients classified as overweight vs. obese (per body mass index [BMI]). This was a retrospective quantitative chart review from three medical weight loss clinics. A de-identified dataset was compiled, consisting of patient demographic data and the genotypes of six specific genes related to six eating behavior traits, or genetically determined characteristics, of eating disinhibition, food desire, hunger, satiety, snacking, and sweet tooth. Each genotype associated with these eating behavior traits was characterized as "typical" (non-weight-promoting behavior) or "altered" (weight-promoting behavior). A total of 768 charts were reviewed; 698 met inclusion criteria. Patients were separated into two groups: $\mathrm{BMI}<30 \mathrm{~kg} / \mathrm{m}^{2}$ and BMI $\geq 30 \mathrm{~kg} / \mathrm{m}^{2}$. Of the six eating behavior traits, a statistically significant difference was noted between the two groups only for eating disinhibition $(p<.001)$, defined as the likelihood to eat more than normal in response to a stimulus such as stress, social environment, tasty foods, etc. Based on these results, the altered genotype of the eating disinhibition gene (more likely to exhibit eating disinhibition) is more prevalent in patients with a BMI $\geq 30 \mathrm{~kg} / \mathrm{m}^{2}$ than those with $\mathrm{BMI}<30 \mathrm{~kg} / \mathrm{m}^{2}$. Results suggested that alteration in the eating disinhibition genotype was associated with obesity. Earlier screening for this genetic alteration may help assess risk for obesity, which can lead to earlier intervention to promote optimal health outcomes and reduce obesity-related illnesses.

\section{P38 \\ Preparing Animal Health Professionals with Knowledge Necessary to Understand Nutrigenetics and Nutrigenomics}

\section{Gustavo A. Mendoza-Fandino ${ }^{1}$, Mónica Duque Quintero', Elsa María Vasquez-Trespalacios ${ }^{2}$ \\ ${ }^{1}$ Department of Animal Health, Corporación Universitaria Remington, Medellin, Colombia; ${ }^{2}$ Shool of Medicine, CES, Medellin, Colombia}

Historically in Colombia, the role of veterinary practitioners has focused on handle and curing productive animals. Our advancing knowledge about animal genomes, now offer the opportunity to individualize dietary intervention to help prevent, mitigate or cure diseases. The development of genomics tools and collecting proof about the constituents of nutrients interacting with the genome as a mechanism to modulate the expression, open opportunities for the advancement of health and disease prevention based on the nutrition. The aim of this work is to describe strategies to introduce nutrigenomics and nutrigenetis topics for Colombian animal health professionals. The first step to achieve this goal was the adding of basic assignment topics related to understand nutrigenetic and nutrigenomics. These topics covered DNA, RNA, proteins, genomic process and regulation looking to a deeper understanding of molecular biology. The introduction of genomics and genetics concepts, in regular courses, facilitate the integration of the new concepts with the classic. Additionally, the animal science department create non-credits study groups, open to all students interested in the topic. The idea with these study groups is to promote abilities of critical thinking and reading of scientific literature related to nutrigenetic and nutrigenomics, looking to promote the animal care based in evidence. The study group has regularly organized talks, with professionals in topics related to animal nutrition and molecular biologist, to generate collaborative projects to involucrate students, as well as to expose them to both areas. Our participation in the 11th Congress of the International Society of Nutrigenetics and Nutrigenomics is our first international effort to be part of the community of scientist that are working to understand and solve the issues related to the nutrition and human health.

P39

\section{A Population-Based Analysis of the Relationship Between Ceramide Levels and Insulin Resistance}

\author{
Frode Norheim ${ }^{1}$, Thomas Bjellaas ${ }^{2}$, Simon T. Hui', \\ Karthickeyan C. Krishnan', Calvin Pan'1, Yehudit Hasin- \\ Brumshtein', Brian W. Parks' ${ }^{3}$ Daniel Y. Li', Jakleen Lee', \\ Sonul Gupta1, Hai H. Bui", Marian Mosier, \\ Stanley L. Hazen", Thomas E. Gundersen², \\ Margarete Mehrabian ${ }^{1}$, W. H. Wilson Tang ${ }^{5}$, \\ Andrea L. Hevener ${ }^{6}$, Christian A. Drevon ${ }^{7}$, Aldons J. Lusis ${ }^{1}$ \\ ${ }^{1}$ Division of Cardiology, Department of Medicine, \\ University of California at Los Angeles, Los Angeles, CA \\ 90095, USA; ${ }^{2}$ Vitas AS, Oslo Innovation Park, \\ Gaustadalleen 21, N-0349 Oslo, Norway; ${ }^{3}$ Department of \\ Nutritional Sciences, University of Wisconsin-Madison, \\ Madison, USA; “Lilly Research Laboratories, Indianapolis, \\ IN, USA; ${ }^{5}$ Department of Cellular and Molecular Medicine, \\ Lerner Research Institute, Cleveland Clinic, Cleveland, \\ USA; ${ }^{6}$ Division of Endocrinology, Diabetes and \\ Hypertension, Department of Medicine, University of \\ California at Los Angeles, Los Angeles, CA 90095, USA; \\ ${ }^{7}$ Department of Nutrition, Institute of Basic Medical \\ Sciences, University of Oslo, Norway
}

Background and Aims: Previous studies have suggested a causal relationship between levels of ceramides and insulin resistance (IR). In particular ceramides derived from C16:0 appear to oppose insulin action most potently. Here, we report a population-based analysis of ceramide metabolism in a panel of genetically diverse inbred strains of mice differing widely in IR 
Methods: Eight weeks old mice were fed a High-Fat, HighSucrose diet for 8 weeks to induce obesity, steatosis and insulin resistance. Liver ceramides from 98 strains of mice of both sexes were measured using mass spectroscopy. We also tested if gonadectomy had an effect on hepatic ceramide accumulation in both sexes of three unique strains of mice $(\mathrm{C} 3 \mathrm{H} / \mathrm{HeJ}, \mathrm{C} 57 \mathrm{BL} / 6 \mathrm{~J}$ and $\mathrm{DBA} / 2 \mathrm{~J})$. The gonadectomized mice were compared to untreated and sham operated controls.

Results: Consistent with previous studies, ceramide levels were significantly associated with IR in male mice, and a similar significant association were observed in female mice. Surprisingly, however, female mice exhibited substantially higher hepatic C16:0 and C18:0 ceramide levels than male mice, despite being less insulin resistant. This also appears to be the case in humans based on levels of ceramides in plasma. We furthermore show that the sex difference in hepatic ceramides is mediated in part by testosterone repression of ceramide synthase 6 .

Conclusions: Our data shows that most female mice strains have higher levels of liver ceramide $\mathrm{C} 16: 0$ and $\mathrm{C} 18: 0$ than their male counterpart. This sex difference can probably be explained by the inhibitory effect of testosterone in male mice. Future mechanistic mice studies will show if liver ceramides oppose insulin action more potently in males than in females.

\section{P40 \\ Is Your Eating Behavior Shaped by Nature or Nurture? A Look into the Nutrigenomics of Taste

\author{
Raquel Novak \\ ${ }^{1}$ Leonard Davis School of Gerontology, University of \\ Southern California, Los Angeles, CA, USA
}

The bitter taste receptor, known as the TAS2R38 gene, has the ability to influence one's nutritional intake due to the perceived intensities of certain foods. A variety of children and adult studies have exposed links to increased sugar, sodium and fat intake or decreased vegetable intake based on participants' perception of bitter taste. Individuals are born as either a taster or non-taster; this genetically inherited profile can affect preference of certain foods, potentially leading to unhealthy food choices and possible chronic diseases. Throughout numerous studies, tasters commonly disliked bitter vegetables and reported a heightened preference of sugar-sweetened items. Tasters experience increased, sometimes unpleasant, sensitivity to bitter compounds, whereas non-tasters experience decreased sensitivity or bland taste to the same bitter compounds. While nontasters consumed a greater variety of dark vegetables, they preferred higher fat foods to provide a satisfying texture and mouth-feel to compensate for the lack of flavor non-tasters may experience. Appropriate nutrition interventions must take place to ensure a balanced, diverse, and nutritious diet for both tasters and non-tasters. The purpose of this article is to expand and increase awareness of genetic involvement with taste preferences and how to implement adequate nutrition interventions. Dietitians can work with both tasters' and non-tasters' genetically pre-disposed flavor-profiles and transform their taste preferences to improve overall quality of life.

\section{P41 \\ Identification of Plasma Metabolites Associated with NAFLD in Morbidly Obese Mexican Subjects}

Elvira Ocampo-Medina', Paola León-Mimila', Joel VegaBadillo', Luis Macías-Kauffer', Hugo Villamil-Ramírez', Blanca López-Contreras', Laura Reyes-Fermín', Francisco Campos-Pérez², Isabel Ibarra-González ${ }^{3}$, Marcela Vela-Amieva ${ }^{3}$, Carlos A. Aguilar-Salinas ${ }^{4}$, Rogelio Hernandez-Pando 5 , Nahum Méndez-Sanchez 6 , Simon Hui ${ }^{7}$, Aldons J. Lusis' ${ }^{7}$, Stanley Hazen ${ }^{8}$, Adriana Huertas-Vazquez', Samuel Canizales-Quinteros ${ }^{1}$

${ }^{1}$ The National Institute of Genomic Medicine, Mexico City; ${ }^{2}$ Clinic for Bariatric Surgery, Hospital Dr. Rubén Leñero, Mexico City; ${ }^{3}$ The National Institute of Pediatrics, Mexico City; ${ }^{4}$ Department of Endocrinology, The National Institute of Medical Sciences and Nutrition, Mexico City; ${ }^{5}$ Department of Pathology, The National Institute of Medical Sciences and Nutrition, Mexico City; ${ }^{6}$ Médica Sur Clinic Foundation, Mexico City; ${ }^{7}$ Department of Medicine, Division of Cardiology, David Geffen School of Medicine, University of California, Los Angeles, USA; ${ }^{8}$ Cleveland Clinic, Cleveland OH, USA

Background: Non-alcoholic fatty liver disease (NAFLD) is a multifactorial disease characterized by an excess of liver fat infiltration and includes a spectrum ranging from steatosis, non-alcoholic steatohepatitis/NASH to cirrhosis and hepatocellular carcinoma. The incidence of steatosis and its progression to NASH varies among different populations, where Mexicans have the highest prevalence. The gold standard for NAFLD is the histopathological evaluation of liver biopsy, an invasive approach that is not broadly applicable to the general population. We applied targeted metabolomics and genome-wide association studies (GWAS) to discover novel biomarkers for NAFLD.

Methods and Results: A total of 46 plasma metabolites including amino acids, TMAO, choline betaine and acyl carnitines were measured using a targeted metabolomic approach. We investigate plasma levels in 425 morbidly obese Mexican subjects who underwent bariatric surgery with liver histology available (without steatosis $(n=41)$, steatosis $(n=88)$, NASH borderline $(n=136)$ and NASH $(n=160)$. We used ANCOVA to compare the differences between the groups adjusting for covariates such as age, sex and BMI. We observed that plasma levels of tyrosine $\left(r=0.27, p=4 \times 10^{-6}\right)$, alanine $\left(\mathrm{r}=0.26, \mathrm{p}=4.7 \times 10^{-6}\right)$, proline $\left(\mathrm{r}=0.22, \mathrm{p}=1.7 \times 10^{-4}\right)$, methionine $(\mathrm{r}=0.17, \mathrm{p}=0.005)$, TMAO $(\mathrm{r}=0.14, \mathrm{p}=0.03)$ and choline $(\mathrm{r}=0.13$, $\mathrm{p}=0.03)$ were associated with NAFLD activity score (NAS) and its components including steatosis, inflammation and hepatocellular ballooning. In addition, we performed a GWAS to identify the genetic factors associated with plasma metabolite levels. We identified 3 SNPs within the proline dehydrogenase $1(P R O D H)$ gene to be significantly associated with proline levels $\left(\mathrm{P}<5 \times 10^{-8}\right)$ and NAS score $(\mathrm{r}=0.83, \mathrm{p}=0.0002)$.

Conclusions: Our findings suggested that plasma metabolites could distinguish between different grades of NAFLD, and could help to determine the mechanisms involved in NAFLD manifestation and progression. 


\section{P42 \\ A Regionalized Genome-Based Mexican Diet Normalizes Hypertriglyceridemia and Improves Insulin Resistance in Subjects with Metabolic Risk Factors for Chronic Disease}

\author{
Claudia Ojeda-Granados $^{1,2}$, Arturo Panduro 1,2, \\ Maricruz Sepúlveda-Villegas ${ }^{1,2}$, Ingrid Rivera-Iñiguez ${ }^{1,2}$, \\ Sonia Roman ${ }^{1,2}$ \\ ${ }^{1}$ Department of Molecular Biology in Medicine, Civil \\ Hospital of Guadalajara, "Fray Antonio Alcalde", \\ Guadalajara, Jalisco, Mexico; ${ }^{2}$ Health Sciences Center, \\ University of Guadalajara, Guadalajara, Jalisco, Mexico
}

Background and Aim: Our preceding analysis of the prevalence profile of polymorphisms in diet-related adaptive genes in West Mexico population suggested that they remain adapted to the features of the low-fat pre-Hispanic diet. Thus, a regionalized genome-based Mexican diet was designed. Herein, we aimed to evaluate its effect on biochemical and anthropometric variables in subjects with metabolic risk factors for chronic disease.

Methods: A quasi-experimental study was performed in 37 eligible mestizo subjects that underwent a 6-month dietary intervention. The diet mainly included available regional Mexican foods, known to have been the staples of the pre-Hispanic diet (e.g. beans, tortilla, tomato, avocado, chia, amaranth, etc.); and macronutrients distribution were $20 \%$ proteins, $50 \%$ carbohydrates, and $30 \%$ fat (of which saturated fat $<7 \%$, monounsaturated fat $10-15 \%$ and polyunsaturated fat $10 \%)$. Anthropometric and biochemical variables were measured at baseline and the changes after 3 and 6 months.

Results: A total of 33 subjects (age $42.6 \pm 11.7 y, 75.8 \%$ female) completed dietary intervention. Metabolic risk factors at baseline were: obesity (body mass index (BMI) of $30.1 \pm 5.6 \mathrm{Kg} / \mathrm{m}^{2}$, body fat excess (BFE) of $15.8 \pm 10.5 \mathrm{Kg}$, insulin resistance determined by a homeostasis model assessment (HOMA-IR) of 3.5 \pm 4.3 , and high serum levels of triglycerides $(151.6 \pm 86.9 \mathrm{mg} / \mathrm{dl})$ and VLDL-c $(31.8 \pm 21.5 \mathrm{mg} / \mathrm{dl})$. Significant changes in anthropometric and biochemical variables were observed after $3 \mathrm{mo}$ and were maintained until the end of the dietary intervention (baseline $v s$. 6mo): weight ( $80.4 \pm 18.5$ vs. $75.0 \pm 16.6 \mathrm{Kg}$, loss of $7 \%$ of their initial weight); BMI (30.1 \pm 5.6 vs. $\left.28.1 \pm 5.1 \mathrm{Kg} / \mathrm{m}^{2}\right)$; BFE $(15.8 \pm 10.4$ vs. $10.5 \pm 10.2 \mathrm{Kg})$; body fat percentage $(35.6 \pm 7.0 v$ s. $31.9 \pm 7.9 \%)$; waist circumference $(95.6 \pm 14.5$ vs. $88.1 \pm 14.0 \mathrm{~cm})$; waist-to-hip ratio $(0.87 \pm 0.08 v s$. $0.84 \pm 0.09)$; fasting plasma glucose $(89.0 \pm 10.5 v s .84 .3 \pm 6.6 \mathrm{mg} / \mathrm{dl})$ and insulin $(14.6 \pm 14.3 v s .9 .0 \pm 5.3)(p<0.05)$. Also, the abnormal values of HOMA-IR (3.5 $\pm 4.3 v s$. 1.9 \pm 1.2$)$, triglycerides (151.6 $\pm 86.9 v s$. $108.5 \pm 44.7 \mathrm{mg} / \mathrm{dl})$ and VLDL-c $(31.8 \pm 21.5 v s .23 .3 \pm 11.3 \mathrm{mg} / \mathrm{dl})$ significantly changed and normalized $(p<0.05)$.

Conclusion: A regionalized genome-based Mexican diet normalized hypertriglyceridemia, HOMA-IR and VLDL-c values, and improved body composition. The recommendation and use of a more traditional diet based on the knowledge of Mexicans cultural history and their diet-related genetic adaptations may be efficient to prevent the development of the current non-communicable chronic diseases.

\section{P43}

The Effect of Selenium and Multiple Micronutrient Administration during Periconceptional Period on The Level sFlt-1

Yustiyanty Monoarfa1, Bambang Wirdjatmanto', Erry Gumilar ${ }^{2}$, Abd. Razak Thaha ${ }^{3}$, Anang S. Otoluwa ${ }^{3}$, Herwin Yatim ${ }^{4}$, Maryam Daafar ${ }^{5}$, Ferlin Monggesang ${ }^{6}$

${ }^{1}$ Department of Nutrition, Faculty of Public Health, Universitas Airlangga, Surabaya, Indonesia; ${ }^{2}$ Department of Fetomaternal, Faculty of Medicine, Soetomo General Hospital, Universitas Airlangga, Surabaya, Indonesia; ${ }^{3}$ Department of Public Health Nutrition, Faculty of Public Health, Universitas Hasanuddin, Makassar, Indonesia; ${ }^{4} \mathrm{PhD}$ Student in Brawijaya Malang, Indonesia; ${ }^{5} \mathrm{Head}$ of Banggai Distict Agriculture and Holticulture Office;

${ }^{6}$ Banggai District Food Security Office, Indonesia

Background/Aims: Early prevention of preeclampsia can be conducted by observing markers of preeclampsia before the occurence of clinical symptoms and signs. The role of Selenium in preventing preeclampsia has been proved in some studies. This study aims to prove the effect of Selenium on women during the period of periconception on Sflt1 level in plasma.

Methods/Design: Study was conducted in three subdistricts in the city of Luwuk, Banggai Regency, from October 2016 to Juny 2017 with double blind randomized controlled trial design. Of 84 preconception women recruited, 12 of them were pregnant and they were randomly assigned into intervention group $(n=7)$, and control group $(n=5)$. The intervention group received Selenium with multi micronutrient (MMN) capsules, and control group was given ironfolic acid (IFA) capsules every day until they were pregnant. Sflt-1 was examinized using Enzyme Linked Immunosorbent Assay (ELISA). The statistical analysis was conducting using Wilcoxon and Mann-Whitney test.

Results: The results showed that average of Sflt-1 increased in both intervention $(4702 \pm 127) p=0.043)$, and control group (5304 \pm 144); $p=0.018$ ), while mean difference between two groups was not significant $(p=0.87)$. This finding suggest that Selenium with MMN supplementation in periconception period can lowered the level of Sflt-1 eventhough it was not significant compare to the IFA.

Conclusions: Selenium with multi micronutrient (MMN) supplementation during periconception period can lowered the level of Sflt-1 to the same extent as suplementation with iron folic acid (IFA). 
P44

\section{Epigenetic Clock Analysis of Diet and Metabolic Markers}

\author{
Austin Quach ${ }^{1}$, Morgan E. Levine ${ }^{1}$, Steve Horvath ${ }^{1,2}$ \\ ${ }^{1}$ Department of Human Genetics, David Geffen School of \\ Medicine, University of California Los Angeles, USA; \\ ${ }^{2}$ Department of Biostatistics, Fielding School of Public \\ Health, University of California Los Angeles, USA
}

Dietary and nutritional factors have been shown to relate to a number of health-related outcomes, yet there is a need for studies that examine their relationship to molecular aging rates. Toward this end, we study the epigenetic clock and other predictors of chronological age based on site-specific DNA methylation; these biomarkers have previously been shown to be independent predictors of all-cause mortality, chronic conditions, and age-related functional decline. We analyze cross-sectional data from 4,173 postmenopausal female participants from the Women's Health Initiative, as well as 402 male and female participants from the Italian cohort study, Invecchiare nel Chianti, examining associations between accelerated epigenetic aging and dietary, cardiometabolic, and lifestyle factors. Epigenetic age acceleration is broadly defined as the epigenetic age left unexplained by chronological age. Here we examine two distinct measures which are based on different sets of CpGs: intrinsic epigenetic age acceleration (IEAA) which is adjusted for variation in blood cell composition, and extrinsic epigenetic age acceleration (EEAA) which explicitly incorporates age-related blood cell counts. These measures can be interpreted as tracking cell-intrinsic aging and immune system aging, respectively. EEAA exhibits significant associations with reported fish $\left(\mathrm{p}=2 \times 10^{-5}\right)$ and alcohol intake $\left(\mathrm{p}=3 \times 10^{-5}\right)$, education $\left(\mathrm{p}=3 \times 10^{-10}\right)$, BMI $\left(\mathrm{p}=2 \times 10^{-8}\right)$, and blood carotenoid levels $\left(\mathrm{p}=2 \times 10^{-9}\right)$-an indicator of fruit and vegetable consumption, whereas IEAA is associated with reported poultry intake $\left(\mathrm{p}=4 \times 10^{-3}\right)$ and BMI $\left(\mathrm{p}=1 \times 10^{-6}\right)$. Both EEAA and IEAA were also found to relate to indicators of metabolic syndrome which appear to mediate their associations with BMI. Finally, longitudinal data suggests that an increase in BMI is associated with increase in both EEAA and IEAA. Overall, this analysis of epigenetic aging in blood suggests that the health benefits of fruit, vegetable, lean meat, and moderate alcohol intake, physical activity, and education, as well as the health risks of obesity and metabolic syndrome might be mediated in part by their anti- and pro-aging effects on the epigenome.

\section{P45}

Nutrigenomic Mechanisms Associated with Pet's Obesity and Diabetes: Use of Cinamomun zeylanicum as Alternative to Their Prevention and Treatment

\author{
Mónica Duque Quintero', Gustavo A. Mendoza-Fandino', \\ Laura Velásquez ${ }^{1}$ \\ ${ }^{1}$ GINVER Research group, Department of Veterinarian \\ Medicine, Corporación Universitaria Remington, Medellin, \\ Colombia
}

The prevalence of overweight and obese pets has increased in recent years. Excess weight is the most common medical condition in companion animals and has a number of health and wellness implications for both pets and their owners. Obese pets also increase health risks, including an increased risk of arthritis, diabetes mellitus, hepatic lipidosis and insulin resistance. Research has shown that the risk for development of diabetes increases about 2-fold in overweight pets and about 4-fold in obese pets. Altered adipokine secretion appears to be an important mechanism for the link between excess BW and many diseases. In addition, adipose tissue is an active producer of hormones, such as resistin, leptin and cytokines, including many inflammatory cytokines such as tumor necrosis factor- $\alpha$, IL-1 $\beta$ and IL-6, and C-reactive protein. The persistent low-grade inflammation secondary to obesity is thought to play a causal role in chronic diseases such as osteoarthritis, cardiovascular disease, diabetes mellitus, and others. For example, tumor necrosis factor- $\alpha$ alters insulin sensitivity by blocking the activation of insulin receptors. In addition, obesity is associated with increased oxidative stress, which also may contribute to obesity related diseases. Management of obesity involves nutritional change as well as behavioral change. The emerging science of Nutrigenomics considers alternative mechanisms by which plant-derived phytochemicals may to influence signaling pathways within cells through of genes expression. Cinnamon (C. zeylanicum) could have beneficial health properties such as anti-inflammatory effects, anti-microbial activity, blood glucose control, decrease the total and LDL cholesterol levels, and reducing cardiovascular disease. Cinnamon potentiates insulin-regulated glucose utilization by enhancing insulin signaling, and it enhances cellular glucose uptake due to the stimulation of the insulin receptor activity by increasing the concentrations of the phosphorylated intracellular protein IRS-1 and the increasing of PI 3-kinase and the GLUT4 transporter. The future of nutrigenomics will be to generate natural alternatives to prevent and treat several diseases, such as dog's obesity, because pets have a special place in the hearts of their human companions. 
P46

\section{Apolipoprotein E Variants and Sex Modify the Action of Dairy Fat Intake on Plasma HDL-Cholesterol and Adiponectin Concentration}

Ethendhar Rajendiran 1, Yongbo She1, Maude TessierGrenier 2,3, Didier Brassard 2,3, Vanu Ramprasath', Iris Gigleux 2,3, Emile Levy 3,4, Angelo Tremblay 2,5, Patrick Couture ${ }^{2,6}$, Benoit Lamarche ${ }^{2,3}$ Peter Eck', Peter J.H. Jones ${ }^{1}$

${ }^{1}$ Richardson Centre for Functional Foods and Nutraceuticals, University of Manitoba; ${ }^{2}$ School of Nutrition, Laval University; ${ }^{3} \mathrm{NAF}$, Laval University; ${ }^{4} \mathrm{CHU}$ Sainte-Justine Research Center; ${ }^{5}$ Department of Kinesiology, Faculty of Medicine, Laval University, Canada

The health benefits of dairy saturated fat (DSF) intake remains unresolved. Apolipoprotein E (APOE) variants modify plasma lipid concentrations (PLC). However, how APOE variants and sex impact the actions of DSF consumption on PLC are not well understood. The present objective was to evaluate effects of APOE (E2E3, E3 and E3E4) variant and sex in altering the effect of DSF intake on PLC. Using a multicenter randomized crossover trial, 92 women and men consumed five isocaloric diets (35\% energy from fats) for 4-wk/4-wk washout between diets that included; (i) cheese, (ii) butter, (iii) monounsaturated fatty acids (MUFA), (iv) polyunsaturated fatty acids (PUFA) and (v) a low-fat, high-carbohydrate (CHO). Results suggested the overall diet/APOE/sex interaction was significant on plasma HDL-C and adiponectin, not on plasma total cholesterol or LDL-C levels. However, HDL-C levels were increased in women as a function of diet and APOE variant-E3E4 with butter $(1.31 \pm 0.05$ $\mathrm{mmol} / \mathrm{l})$ intake vs men-E3E4 with cheese $(1.02 \pm 0.06 \mathrm{mmol} / \mathrm{p}=$ $0.0265), \mathrm{CHO}(0.98 \pm 0.05 \mathrm{mmol} / \mathrm{l}, \mathrm{p}=0.0 .0041)$, MUFA $(0.99 \pm$ $0.06 \mathrm{mmol} / \mathrm{l}, \mathrm{p}=0.00065)$ and PUFA $(0.95 \pm 0.06 \mathrm{mmol} / \mathrm{l}, \mathrm{p}=0.0011)$. Further, increased HDL-C was observed in women-E3E4 with cheese vs men-E3E4 with PUFA $(1.30 \pm 0.05 \mathrm{mmol} / \mathrm{l}$ vs $0.99 \pm 0.06 \mathrm{mmol} / \mathrm{l}$, $\mathrm{p}=0.0032)$. Moreover, adiponectin levels were elevated in womenE3 with butter $(7.82 \pm 0.49 \mu \mathrm{g} / \mathrm{l})$ intake vs men-E3 with cheese $(5.11$ $\pm 0.47 \mu \mathrm{g} / \mathrm{l}, \mathrm{p}=0.0224)$, CHO $(5.01 \pm 0.47 \mu \mathrm{g} / \mathrm{l}, \mathrm{p}=0.0126)$, MUFA $(5.28 \pm 0.46 \mu \mathrm{g} / \mathrm{l}, \mathrm{p}=0.0534)$, PUFA $(5.17 \pm 0.47 \mu \mathrm{g} / \mathrm{l}, \mathrm{p}=0.0317)$ intakes. Also, women-E3E4 with butter and cheese intake showed high levels of adiponectin compared to CHO, MUFA and PUFA (not shown here). Our findings demonstrate the importance of evaluating APOE variants and sex effect among the diet interaction related to cardiometabolic risk (CMR). However, DSF from either butter or cheese seems to have a beneficial effect on plasma HDL-C and adiponectin levels in women with APOE-E3E4 variant compared to men with APOE-E3E4 variant compared to other fat intake. In summary, APOE variants and sex impact the role of DSF from the dairy on other CMR factors.

\section{P47}

Assessment of Mercury and Lead Content of Eight Fish Species from the Colombian Caribbean

\section{Claudia Ramírez}

Faculty of Health Sciences, Nutrition and Dietetics, Corporación Universitaria Remington, Medellín, Colombia

Human fish consumption provides nutrients like protein, omega-3 and omega-6, polyunsaturated fatty acids, minerals, and vitamins, as well as xenobiotics, such as heavy metals coming from natural and anthropogenic pollution that affects aquatic ecosystems and, therefore, phytoplankton, zooplankton, fish, and ultimately humans. Through the aquatic food chain, there is bioaccumulation and biomagnification of heavy metals, such as mercury; therefore, this effect is greater in carnivorous fish than in herbivorous. Mercury, under its methylmercury speciation, and lead may go through the blood-brain barrier due to the high specificity of thiol ligands sulfur atoms that enables entry to the endothelial cells causing disorders at the central nervous system level due to its teratogenicity with the possibility to unleash neurotoxicity and alterations, such as microcephalia, cognitive disability, autism, attention deficit, palsy, and physical disability in the fetus, derived from the loss of neuronal cells in specific regions of the brain. Methylmercury genotoxic effects surpass lead because of the high generation of free radicals that unleash oxidative stress and have direct influence on DNA and its repair mechanisms. A research was conducted to determine mercury and lead concentrations to eight fish species of the Colombian Caribbean Sea, using microwave plasma atomic emission spectrometry. Highest mercury concentrations were found in mackerel and shad, with values higher than the allowable maximum limits, according to the Ministery of Health and Social Protection of Colombia and the European Community, which may be explained because they are large carnivorous fish that tend to accumulate muscular fat, to which can be more easily adhere heavy metals. All studied fish species had lead levels higher than the allowable maximum limits, in addition to its nephrotoxic effects. Taking into account the Codex Alimentarius tolerable weekly consumption, safe consumption may be calculated for the different vulnerable groups. 


\section{P48 \\ Association of Gly482Ser PPARGC1A Gene Variant with Differential Cholesterol Outcomes in Response to Two Energy- Restricted Diets Among Subjects with Excessive Weight}

O. Ramos-López'1, J.I. Riezu-Boj'1,2, FI. Milagro 1,3, L. Goni', M. Cuervo ${ }^{1,2,3}$, J.A. Martínez ${ }^{1,2,3,4}$

${ }^{1}$ Centre for Nutrition Research/Department of Nutrition, Food Sciences and Physiology, University of Navarra, Pamplona, Spain; ${ }^{2}$ Navarra Institute for Health Research (IdiSNA), Pamplona, Spain; ${ }^{3}$ CIBERobn, Centro de Investigación Biomédica en Red de la Fisiopatología de la Obesidad y Nutrición. Carlos III Health Institute, Madrid, Spain; ${ }^{4}$ Madrid Institute of Advanced Studies (IMDEA Food), Madrid, Spain

Background: Precision personalized dietary strategies based on nutrigenetics involves the understanding of individual reactions to different treatments depending on the genetic make-up.

Objective: To investigate the influence of two PPARGC1A gene polymorphisms on metabolic outcomes in response to two energyrestricted diets.

Methods: A four-month nutritional intervention was conducted involving two different hypocaloric diets ( $-30 \%$ of energy), based on a low-fat (LF) and on a moderately high-protein (MHP) dietary patterns. Unrelated subjects with overweight and obesity were genotyped for two PPARGC1A polymorphisms: rs8192678 (Gly482Ser) and rs3755863 $(\mathrm{G}>\mathrm{A})$. Genotyping was performed by next-generation sequencing and haplotypes were phenotypically screened. Anthropometric measurements and standard biochemical tests were assessed by validated methods.

Results: Differential cholesterol outcomes were found according to diets and Gly482Ser genotypes. Interestingly, Gly482Gly homozygotes following a LF diet had lower reductions in total cholesterol (-9 $\mathrm{mg} / \mathrm{dL}$ vs. $-27 \mathrm{mg} / \mathrm{dL}, \mathrm{p}=0.017)$ and LDL-c levels $(-5 \mathrm{mg} / \mathrm{dL}$ vs. -18 $\mathrm{mg} / \mathrm{dL}, \mathrm{p}=0.016)$ than 482 Ser allele carriers. However, this finding was not evidenced in the MHP group, where Gly482Gly homozygotes underwent similar cholesterol decreases than 482Ser allele carriers. Likewise, all genotype carriers had significant reductions in the frequencies of hypercholesterolemia (total cholesterol $\geq 200 \mathrm{mg} / \mathrm{dL}$ ), except Gly482Gly homozygotes in the LF group. Meanwhile, the rs3755863 polymorphism and PPARGC1A haplotypes showed borderline effects regarding cholesterol decreases.

Conclusions: An energy-restricted MHP diet might be more beneficial than a LF diet to reduce serum cholesterol among PPARGC1A Gly482Gly genotype carriers. The analysis of this genetic variant might be the basis for a precise nutrigenetic management of hypercholesterolemia based on the genetic make-up.

\section{P49}

\section{Polymorphism in ANKK1/DRD2 Gene Associates with Unhealthy Eating Behaviors and Metabolic Disturbances}

\author{
Ingrid Rivera-Iñiguez ${ }^{1,2}$, Maricruz Sepulveda-Villegas ${ }^{1,2}$, \\ Claudia Ojeda-Granados ${ }^{1,2}$, Sonia Roman ${ }^{1,2}$, \\ Arturo Panduro ${ }^{1,2}$ \\ ${ }^{1}$ Department of Molecular Biology in Medicine, Civil \\ Hospital of Guadalajara, "Fray Antonio Alcalde"; ${ }^{2}$ Health \\ Sciences Centre, University of Guadalajara, Mexico
}

Background: Unhealthy food behaviors affect weight control management. Environmental, social and genetic factors contribute to food decision-making at the brain reward system. ANKK1/DRD2 TaqI A1 (rs1800497) polymorphism affects receptor density while increasing L-DOPA activity in the brain reward system. This polymorphism has been associated with unhealthy behaviors such as gambling, alcohol abuse and substance dependence. A1 allele has been related to weight gain. However little is known about the influence of ANKK1/DRD2 TaqI A1 on dietary quality.

Objective: To identify the association of ANKK1/DRD2 TaqA1 (rs1800497) with unhealthy food intake in West Mexico.

Methods: A cross-sectional study, with 549 subjects from West Mexico was analyzed for ANKK1/DRD2 TaqI A1 genotypes (A1/A1, A1/A2, A2/A2) by an allelic discrimination assay. Anthropometric evaluation, biochemical tests, a $24 \mathrm{hr}$ dietary recall and a food frequency questionnaire, were performed.

Results: Subjects were $37.04 \pm 13.19$ years old. Genotype frequency distribution in West Mexico was A1/A1 (17.3\%), A1/A2 (46.6\%), and A2/A2 (36.1\%). BMI was similar regardless ANKK1/ $\mathrm{DRD} 2$ genotypes $(\mathrm{p}=0.695)$. Triglycerides levels were significantly higher among the A1/A1 genotype $(p=0.047)$. Energy intake did not differ by genotypes $(\mathrm{p}=0.884)$. The $\mathrm{A} 1 / \mathrm{A} 2$ genotype conferred greater risk for lower frequency consumption of legumes and vegetables than $\mathrm{A} 1 / \mathrm{A} 1$ and $\mathrm{A} 2 / \mathrm{A} 2$ genotypes $(\mathrm{OR}=1.73,95 \% \mathrm{CI} 1.03-2.90), \mathrm{p}=$ 0.037 ; and $\mathrm{OR}=2.24,95 \% \mathrm{CI} 1.39-3.58), \mathrm{p}=0.008$ respectively). The A1/A2 genotype conferred increased risk for higher frequency consumption of meats and fats than A1/A1 and A2/A2 genotypes (OR $=1.88,95 \%$ CI $1.15-3.11), \mathrm{p}=0.016$; and $\mathrm{OR}=2.65,95 \% \mathrm{CI} 1.32-$ 5.32), $\mathrm{p}=0.006$ respectively).

Conclusions: Even though energy intake and BMI did not differ by genotypes, the TaqI A1 (rs1800497) polymorphism could be a risk factor for detrimental dietary quality by inducing unhealthy food behaviors that may predispose to metabolic disturbances. 
P50

Folic Acid and Other Methyl Donors Attenuate LPS-Induced Gene Expression of Proinflammatory Cytokines in Human Macrophages

M. Samblas ${ }^{1}$, F.I. Milagro ${ }^{1,2}$, M.A. Zulet ${ }^{1,2,3}$,

${ }^{1}$ Centre for Nutrition Research/Department of Nutrition, Food Sciences and Physiology. University of Navarra, Pamplona, Spain; ${ }^{2}$ CIBERobn, Centro de Investigación Biomédica en Red de la Fisiopatología de la Obesidad y Nutrición. Carlos III Health Institute, Madrid, Spain; ${ }^{3}$ Navarra Institute for Health Research (IdiSNA), Pamplona, Spain; ${ }^{4}$ Madrid Institute of Advanced Studies (IMDEA Food), Madrid, Spain

Background and Objectives: Methyl donors participate in the epigenetics mechanisms by modulating the methylation levels of DNA and proteins. Moreover, methylating compounds trigger essential roles in metabolism and cellular homeostasis. Dietary supplementation with folic acid and other methyl donors has been reported to elicit beneficial effects on obesity-related manifestations and cardiovascular diseases. The aim of this study was to investigate the effects of methyl donors on the inflammatory process in the context of obesity.

Methods: White blood cells and plasma samples were obtained from 97 obese subjects (mean $\mathrm{BMI}=35.8 \pm 4.7 \mathrm{~kg} / \mathrm{m}^{2}$ ) that participated in the Metabolic Syndrome Reduction in Navarra (RESMENA) controlled trial. Methyl donor levels (folic acid and vitamin $\mathrm{B}_{12}$ ) and inflammatory markers were quantified in plasma. Expression levels of inflammation-related genes were quantified in white blood cells. THP-1 cells (human monocytes) were differentiated into macrophages with $25 \mathrm{ng} / \mathrm{ml}$ of phorbol 12-myristate 13-acetate (TPA) and stimulated with $100 \mu \mathrm{g} / \mathrm{ml}$ of lipopolysaccharide (LPS), followed by incubation with $11 \mu \mathrm{M}$ folic acid or a mix of methyl donors (folic acid $11 \mu \mathrm{M}$, choline $100 \mu \mathrm{M}$, and vitamin $\mathrm{B}_{12} 18 \mathrm{nM}$ ) for $24 \mathrm{~h}$.

Results: Inverse correlations were found between plasma folic acid and vitamin $\mathrm{B}_{12}$ concentrations, with TNFa and SERPINE1 expression in white blood cells, as well as with circulating PAI-1 levels. Incubation of THP-1 activated macrophages with folic acid or a mix of methyl donors inhibited significantly ILIB, TNF $\alpha$, SERPINE1 and TLR4 gene expression. Moreover, methyl donors decreased IL1B protein levels.

Conclusions: This study reveals a negative association between methyl donor levels in blood and important inflammatory markers. Moreover, the addition of folic acid or a mix of methyl donors (folic acid, choline, and vitamin $B_{12}$ ) to human macrophages activated with LPS reduced inflammatory gene expression, suggesting a role of methyl donors in obesity-related inflammation.
P51

\section{Caffeine Consumption and Disease Risk Modulated by Genetic Variants in Caffeine Metabolism}

\author{
Maria Schellenberger ${ }^{1}$ \\ ${ }^{1}$ University of Southern California,USA
}

There are many chronic conditions which have been linked with caffeine consumption, including positive associations with hypertension and impaired glucose control, and negative associations with Parkinson's disease and certain cancers. Caffeine is found in a variety of commonly consumed food products including coffee, soft drinks, tea, energy drinks, caffeinated sports drinks, cocoa, chocolate, dietary supplements, and certain medications. Many enzymes are involved in caffeine metabolism, but between $90-95 \%$ is metabolized by cytochrome P450 1A2 (CYP1A2). One SNP of particular importance in influencing the inducibility of CYP1A2 is Rs762551 (aka -164A $>C$ or $-163 \mathrm{C}>\mathrm{A}$ ). For this review, 10 studies were consulted to investigate the impacts of caffeine consumption, genetic variation, and disease risk. Findings indicate there may be varying risks of chronic diseases associated with the SNP CYP1A2, however the risks and benefits are not consistent for each genetic variation. There appears to be a slight increased protective effect of caffeine consumption on Parkinson's disease among slow metabolizers. However, research suggests that slow metabolizers with high caffeine consumption are at increased risk for MI and impaired fasting glucose. Fast metabolizers, on the other hand, may benefit from protective effects of caffeine in preventing hypertension and enjoy higher degrees of ergogenic effects, yet may be at higher risk for bone mineral density loss with increased caffeine consumption. The knowledge of this particular SNP may assist the patient if they are at higher risk for a specific chronic condition (ie diabetes, hypertension, osteoporosis) and are interested in reducing their risk for one specific condition. Due to the various degrees of risk and benefits between these chronic conditions for fast and slow metabolizers, it is difficult to make generalized suggestions for patients regarding their caffeine consumption. 
P52

\section{A Strategy for Discovery of Endocrine Interactions with Application to Whole-Body Metabolism}

Marcus M. Seldin', Simon Koplev², Prashant Rajbhandari', Laurent Vergnes ${ }^{3}$, Prashant Rajbhandari, Gregory M. Rosenberg1, Raffi Gharakhanian', Nam Che 1, Calvin Pan 1, 3 Selina Mäkinen ${ }^{5}$, Yonghong Meng ${ }^{1}$, Diana M. Shih', Mete Civelek ${ }^{6}$, Brian W. Parks ${ }^{7}$, Eric D. Kim', Frode Norheim ${ }^{8}$, Karthickeyan Chella Krishnan ${ }^{1}$, Yehudit Hasin-Brumshtein', Margarete Mehrabian', Markku Laakso', Christian A. Drevon ${ }^{8}$, Heikki A. Koistinen ${ }^{5}$, Peter Tontonoz ${ }^{1}$, Karen Reue ${ }^{3}$, Rita M. Cantor ${ }^{3}$, Johan L.M. Björkegren², Aldons J. Lusis' 1, 3,4,*

'Department of Medicine, ${ }^{3}$ Human Genetics, ${ }^{4}$ Microbiology, Immunology and Molecular Genetics at the University of California, Los Angeles, CA; ${ }^{2}$ Department of Genetics and Genomic Sciences, The Icahn Institute for Genomics and Multiscale Biology Icahn School of Medicine at Mount Sinai, New York, NY; ${ }^{5}$ Department of Medicine, University of Helsinki and Helsinki University Central Hospital, Helsinki, Finland; Minerva Foundation Institute for Medical Research, Biomedicum 2U, Helsinki, Finland, ${ }^{6}$ Department of Biomedical Engineering, University of Virginia, Charlottesville, VA; ${ }^{7}$ Department of Nutritional Sciences, University of Wisconsin, Madison, WI; ${ }^{8}$ Department of Nutrition, Institute of Basic Medical Sciences, Faculty of Medicine, University of Oslo, Oslo, Norway; ${ }^{9}$ nstitute of Clinical Medicine, Internal Medicine, University of Eastern Finland and Kuopio University Hospital, Kuopio, Finland

Inter-tissue communication via secreted proteins has been established as a vital mechanism for proper physiologic homeostasis. We have developed a bioinformatics framework using a mouse reference population, the Hybrid Mouse Diversity Panel (HMDP), which integrates multi-tissue expression arrays and publicly available resources to identify and functionally annotate novel tissue-tissue communication. We validate this method by showing that we can identify known, as well as novel endocrine factors responsible for communication between tissues. We further show the utility of this approach by identification and mechanistic characterization of two new endocrine factors. Adipose-derived Lipocalin-5 is shown to enhance skeletal muscle mitochondrial function and liver-secreted Notum acts to promote white adipose tissue "beiging". We have explored these factors in both basal and high-fat/high-sucrose dietary perturbations and show the physiologic effects are robust and significant. We have additionally screened mouse and human datasets using this method and uncovered in vivo evidence for 7 novel inter-tissue communicatory axes. The developed methodology is generally applicable to any collection of organs, tissues, or cell types sampled from a genetically diverse population and profiled using omics platforms.
P53

Association of the FTO Polymorphism
rs9939609 with Hypertriglyceridemia Is
Independent of Dietary Intake

Maricruz Sepulveda-Villegas ${ }^{1,2}$, Ingrid Rivera-Iñiguez ${ }^{1,2}$, Karina Gonzalez-Aldaco ${ }^{1,2}$, Claudia Ojeda-Granados ${ }^{1,2}$, Sonia Roman ${ }^{1,2}$, Arturo Panduro ${ }^{1,2}$

${ }^{1}$ Department of Molecular Biology in Medicine, Civil Hospital of Guadalajara, "Fray Antonio Alcalde"; ${ }^{2}$ Health Sciences Centre, University of Guadalajara, Mexico

Background: Worldwide, the FTO gene has been associated with obesity mainly in Caucasian populations. Mexico, is an admixed population containing a heterogenic proportion of Amerindian, European and African ancestries and with a high prevalence of obesity. However, the impact of the FTO gene may have on obesityrelated metabolic parameters among Mexican population is not clear.

Objective: To analyze the association of the FTO polymorphism (rs9939609) with dietary intake and metabolic profile in West Mexicans.

Methods: In a cross-sectional study, 333 unrelated subjects $\geq 18$ years old and body mass index (BMI) $\geq 18.5 \mathrm{~kg} / \mathrm{m}^{2}$ from West Mexico were enrolled. Genotyping was performed by an allelic discrimination assay. Biochemical and anthropometric profiles were evaluated by a dry chemistry assay. Food frequency questionnaire and 24-hour dietary recall were performed to assess dietary intake.

Results: The average age of subjects was $36.4 \pm 12.9$ years and BMI of $28.3 \pm 7.9 \mathrm{~kg} / \mathrm{m}^{2}$. Genotype frequencies were AA (8\%), AT $(36 \%)$, and TT $(56 \%)$. Higher levels of insulin, triglycerides and VLDL-c were observed in the TT than AA+AT carriers $(\mathrm{p}<0.05)$. The TT genotype conferred a higher risk for hypertriglyceridemia (HTG) in comparison with the $\mathrm{AA}+\mathrm{AT}$ genotypes $(\mathrm{OR}=1.78,95 \% \mathrm{CI} 1.12$ $2.82, \mathrm{p}=0.016$ ). No differences in energy and macronutrient intake were observed among genotypes. Stratification by BMI showed that normal weight subjects with TT genotype maintained higher levels of triglycerides, and VLDL-c than AA+AT genotypes $(p<0.05)$. In the same group those with the TT genotype had higher risk for HTG $(\mathrm{OR}=2.58,95 \% \mathrm{CI} 1.13-5.89, \mathrm{p}=0.017)$. Besides, in the obese group, TT carriers had higher levels of insulin, HOMA-IR, triglycerides, and VLDL-c in comparison with AA+AT $(\mathrm{p}<0.05)$. Hypertriglyceridemia was not related to any dietary feature by genotypes.

Conclusions: The TT genotype of FTO was associated with HTG, independently of dietary intake in West Mexicans. This could be explained because, the AA genotype may promote higher adiposity, increased triglyceride uptake and obesity, whereas the TT genotype could have a lower adipose capacity resulting in high circulating triglycerides in Mexicans. However, further research is needed. 


\section{P54}

\section{Whole Egg Consumption Does Not Alter Expression of Genes Related to Cholesterol Homeostasis or Inflammation in PBMC of Overweight, Postmenopausal Women}

\author{
Allison M. Stevens ${ }^{1}$, Lisa R. Sawrey-Kubicek', \\ Chenghao Zhu', Jody M. Randolph'1, Angela M. Zivkovic', \\ Francene M. Steinberg ${ }^{1}$ \\ ${ }^{1}$ Department of Nutrition, University of California, \\ Davis, CA, USA
}

Introduction: Eggs yolks are rich sources of lipids including cholesterol, ceramides, sphingolipids, and phospholipids. These bioactive compounds have been shown to impact the lipidome and proteome of lipoproteins, potentially altering cholesterol homeostasis and inflammation in the body. We investigated whether consumption of two whole eggs for four weeks altered expression of genes related to cholesterol flux and inflammation in peripheral blood mononuclear cells (PBMC) in overweight, postmenopausal women.

Methods: Using a randomized, controlled, crossover design, 20 participants (BMI $28.4 \pm 2.9$ ) were randomized to consume either a daily breakfast containing two whole eggs or the equivalent amount of egg whites for four weeks separated by a four-week washout period. PBMC were isolated from fasted blood before and after each dietary intervention for RNA extraction. Targeted gene expression analyses were assessed using qPCR.

Results: Expression of genes related to cholesterol synthesis and transport in PBMC including 3-hydroxy-3-methylglutaryl-CoA reductase $(H M G C R)$, ATP binding cassette subfamily A member 1 $(A B C A 1)$, ATP binding cassette subfamily $\mathrm{G}$ member 1 ( $A B G C 1)$, and ADP ribosylation factor like GTPase $4 \mathrm{C}(A R L 4 C)$ did not differ between whole egg and egg white interventions. Higher PBMC expression levels of pro-inflammatory cytokines tumor necrosis factor $(T N F)$ and interleukin 1 beta $(I L 1 B)$ were observed following the egg white intervention compared with whole egg, however these were not statistically significant $(\mathrm{p}=0.06$ and $\mathrm{p}=0.12$, respectively). Similarly, toll like receptor 4 (TLR4) expression levels did not differ between interventions.

Conclusions: Expression of genes related to cholesterol homeostasis in PBMC were unaffected by consumption of two eggs per day for four weeks in postmenopausal women. ILIB and TNF demonstrated more responsiveness to the dietary intervention with trends toward increased expression during the egg white intervention versus the whole egg intervention, however these changes were not statistically significant.
P55

\section{Can the Intestinal Microbiome Enhance the Protective Effect of Calcium and Fiber on Colorectal Cancer?}

\author{
Leah T. Stiemsma ${ }^{1}$, Karin B. Michels
}

${ }^{1}$ Department of Epidemiology, Jonathan and Karin Fielding School of Public Health, University of California, Los

Angeles, USA

Colorectal cancer (CRC) is the second leading cause of cancerrelated deaths in the US. Epidemiologic research supports a protective effect of calcium $(\mathrm{Ca}+)$ consumption on CRC development. Further, the protection of $\mathrm{Ca}+$ is increased when combined with dietary fiber and several human and mouse studies have suggested that dietary fiber increases $\mathrm{Ca}+$ absorption. However, the underlying mechanism of this association with CRC protection remains unclear. One mechanistic explanation might be related to intestinal permeability, a risk marker for CRC, which is associated with variations in the gut microbiome composition and function. Most of the mechanistic work in this area has been conducted in animal models and clinical human studies are lacking. Here we describe a pilot randomized crossover intervention study comprised of 4 men and 4 women aged 20 - 35 years, established to address the following hypothesis: Fiber amplifies $\mathrm{Ca}+$ absorption to decrease intestinal permeability through perturbations to the intestinal microbiome. Our intervention includes 4 randomized trial periods: (1) $\mathrm{Ca}+$ powder, (2) soluble corn fiber (SCF), (3) combination of $\mathrm{Ca}+$ and SCF, and (4) placebo. Stool and blood samples will be collected at baseline and during the last day of each intervention and each washout period. We will analyze the gut microbiome composition using 16S ribosomal RNA gene sequencing. The concentration of circulating lipopolysaccharide (LPS, via Limulus Amebocyte Lysate Assay) will serve as a measure of intestinal permeability. This pilot project will allow us to gather valuable information on the intestinal microenvironment and the systemic effects associated with these dietary components, which will guide our future study design in a larger cohort. The results from our work may provide an easily translatable, feasible, and inexpensive way to contribute to the prevention of CRC.

\section{P56 \\ MTHFR Polymorphism C677T May Modify Colorectal Cancer}

\author{
Whitney English Tabaie ${ }^{1}$ \\ ${ }^{1}$ Davis School of Gerontology, University of Southern \\ California, USA
}

Background: The B vitamin folate participates in two critical metabolic cycles in the body involved in DNA synthesis, repair, and methylation. One of the cycles, known as the methionine-homocysteine cycle, occurs inside of cells with folate acting as a methyl donor in the conversion of homocysteine to methionine. The reaction is catalyzed by the enzyme methylenetetrahydrofolate reductase (MTHFR). MTHFR reduces 5,10-methylenetetrahydrofolate to 
5-methyltetrahydrofolate. Individuals with mutations in the MTHFR gene have impaired folate metabolism, which is associated with hyperhomocysteinemia, DNA strand breaks, and aberrant DNA methylation. Imbalanced DNA-methylation is commonly associated with colorectal cancer (CRC), however, studies suggest that the MTHFR mutation may actually provide protection against the disease by increasing the availability of 5,10-methylenetetrahydrofolate, which aids in nucleotide synthesis and decreases DNA breakage. This review examines the available literature on this complicated connection.

Methods: Web of Science and PubMed databases were searched using the terms "MTHFR mutation and colorectal cancer" and "rs1801133 and colorectal cancer." Ten studies were selected, including one cohort study, three case-control studies, and six meta-analyses.

Results: Of the six meta-analyses, four found significant inverse associations between TT/CT genotypes and CRC risk compared to the $\mathrm{CC}$ genotype, one found a small significant positive association between the TT genotype and CRC risk, and one found no association. All three of the case-control studies found an inverse risk of $\mathrm{CRC}$ for individuals with the TT mutation. However, the cohort study observed an increased risk of mortality for individuals with CRC who have a homozygous mutation (TT, HR: 2.50) as well as those with a heterozygous mutation (CT, HR: 1.85).

Conclusion: Folate likely plays a dose and time dependent role in the development of CRC and may modify the effects of MTHFR mutations. More research is needed on the association between mutations and CRC, taking into account folate status, along with other factors involved in folate metabolism including vitamin B2, B12, and homocysteine.

P57

\section{Cardiovascular Risk in CKD Patients Affected by Arterial Calcifications (AC)}

Annalisa Terranegra', Massimo Mezzavilla', Sara Tomei", Lee Silcock', Caterina Brasacchio ${ }^{2}$, Laura Soldati², Teresa Arcidiacono ${ }^{3}$, Lorenza Macrina ${ }^{3}$, Giuseppe Vezzoli3.

${ }^{1}$ Sidra Medical and Research Center, Qatar; ${ }^{2}$ Università degli Studi di Milano, Italy; ${ }^{3}$ Ospedale San Raffaele, Milan, Italy

Chronic Kidney Disease (CKD) patients at different stages suffer of high risk of cardiovascular mortality that is higher in dialysis patients than in general population. The current study recruited 208 CKD patients in hemodialysis and collected dietary $24 \mathrm{hrs}$ recalls, medical history, biochemical parameters and blood samples for genetic analyses. The main aim is to identify genetic and nutritional factors which could increase the risk of cardiovascular events in CKD. SNPs genotyping was performed using Illumina ${ }^{\circledR}$ MegaEX Array (2M SNPs) comparing patients with previous cardiovascular events (CV1; 135 subjects) vs patients without cardiovascular events (CV0; 73 subjects). For each marker we calculated the Fixation Index (Fst) using the CV1 and CV0 as subpopulations in order to identify the most differentiated SNPs between the two subgroups. Using the cut-off of the $99^{\text {th }}$ percentile of the Fst distribution we identified 3218
SNPs as differentiated between CV1 and CV0. We interrogated two functional pathway tools (Panther and Ingenuity Pathway Analysis IPA) to define any possible biological/pathological role of the associated SNPs. We merged the most significant results from the two tools and we identified two genes involved in both cardiovascular disease pathways (from IPA) and hearth development (Panther): Glucagonlike peptide-1 receptor (GLP1R) and Sarcoglycan delta (SGCD). Using regression tree analyses we found that GLP1R rs10305445 minor allele $A$ is associated with higher percentages of $\mathrm{CV} 1(\mathrm{p}=0.03)$ and, inversely, SGCD rs145292439 minor allele A is associated with lower percentages of CV1 $(p=0.038)$ and higher levels of HDL $(p=0.015)$. We did not find any significant difference in the dietary intake between the two groups as well as with the genetic data and the biochemical parameters. From this preliminary analysis, we can conclude that the genetic factors may contribute more than diet to cardiovascular disease in CKD patients and we can speculate a role of genetic risk marker for GLP1R as well as a protective marker for SGCD in CKD.

\section{P58 \\ Nutrigenetic Approach: Does Vitamin D Status, CYP27B1 rs 10877012 and Its mRNA Levels Influence Essential Hypertension in Indian Population?

\author{
Varsha Varakantham ${ }^{1}$, Ashok Kumar Kurakula Sailoo², \\ Balakrishna Nagalla ${ }^{3}$, Dinesh Kumar Bharatraj ${ }^{1}$ \\ ${ }^{1}$ Food and Drug Toxicology Research Centre, National \\ Department, Department of General Medicine, Gandhi \\ Hospital, Secunderabad, India; ${ }^{3}$ Department of \\ Biostatistics, National Institute of Nutrition, Hyderabad, \\ India
} Institute of Nutrition, Hyderabad, India; ${ }^{2}$ Former Head of}

CYP27B1 (25-OH vitamin D $1 \alpha$ hydroxylase) is the rate-limiting enzyme for bioactivation of $1,25(\mathrm{OH})_{2}$ vitamin $\mathrm{D}$ from $25(\mathrm{OH})$ vitamin $\mathrm{D}$, and it is well known that low levels of vitamin $\mathrm{D}$ can lead to increased renal renin production, thus activating the renin-angiotensin-aldosterone system, thereby increasing blood pressure. Therefore, we have conducted a preliminary investigation in 292 hypertensives and 324 controls to evaluate the association of the genetic variant of CYP27B1 rs10877012, its' mRNA expression and 25(OH) vitamin D in Indian population with essential hypertension. The percentage of rs $10877012 \mathrm{GG}, \mathrm{GT}$ and TT in controls are $19.8 \%, 54.3 \%, 25.9 \%$ and in hypertensives $21.9 \%, 50.7 \%, 27.4 \%$ respectively. It is interesting to note that, we determined $\mathrm{T}$ (Allele frequency $=0.53$ ) as the major allele, whereas it is found to be the minor allele in all the studies done so far in various other populations. The highest allele frequency till now for T allele (0.49) was observed in Bengali in Bangladesh (BEB) population derived from 1000 genome project phase 3 . In multivariate logistic analysis after adjustment for covariates including age, gender and body mass index (BMI), no association between the CYP27B1 rs10877012 gene variants and essential hypertension was seen ( $p>0.05$ ). Only $6.4 \%$ and $3.7 \% 25(\mathrm{OH})$ Vitamin D sufficiency $(\geq 30 \mathrm{ng} / \mathrm{mL}$ ) was observed in hypertensives and controls, although India has abundant sunlight. In addition, we found no association of 
25(OH) Vitamin D status with essential hypertension (controls: $14.1 \pm 8.2 \mathrm{ng} / \mathrm{mL}$ versus cases: $13.9 \pm 7.5 \mathrm{ng} / \mathrm{mL}, \mathrm{p}>0.05$ ). Even the mRNA expression levels, failed to show any differences based on the genetic polymorphism between hypertensives and controls. In conclusion, these results clearly suggest that Vitamin D status and CYP27B1 genetic variants may not play any significant role in disease susceptibility towards hypertension in Indian population. Further, robust nutrigenetics studies including more number of genes of Vitamin D metabolic pathway and renin-angiotensin-aldosterone system with higher sample size are warranted to draw specific conclusions. kinase, whose mediator in cyclic GMP. These changes are accompanied by altered transcription and epigenetic mechanisms, and are reversed by augmenting the cellular energy charge through supplementation of the corresponding deficient cofactor. The effects are associated with parallel modifications in the corresponding metabolic pathways. They are associated with muscular insulin sensitivity modifications mediated directly by AMPK and the glucose transporter GLUT4. We propose that privation and supplementation of OXPHOS cofactors are analogous to fasting and feeding, and thus are models to better understand the mechanisms underlying obesity and diabetes after excesive alimentation and life style changes.

\section{P59 \\ The Mexican Roots of Nutrigenetics and Nutrigenomics}

\author{
Antonio Velazquez-Arellano ${ }^{1}$, Hector Bourges-Rodriguez ${ }^{2}$ \\ ${ }^{1}$ Nutritional Genetics Unit, Institute of Biomedical \\ Investigations UNAM and ${ }^{2}$ National Institute of Medical \\ Sciences and Nutrition "Salvador Zubiran", Mexico City, \\ Mexico
}

It is surprising that until just three or four decades' ago, collaborations between nutritionists and geneticists were minimal. The developments of molecular biology and of genomics have blurred this false dichotomy. We put forward a historical perspective on this conceptual transformation, that may be considered as revolutionary, and show that some of its origins emerged in Mexico during the 1980s. This presentation focuses on the Genetic Factors in Nutrition workshop in Mexico in 1982 and the ensuing book published in 1984. Further Mexican and international developments are briefly mentioned.

\section{P60 \\ Cellular Energy Status Affects Carbon Metabolism Gene Expression and Has Metabolic and Possible Clinical Consequences}

Alain de J. Hernandez-Vazquez', Josue Andres GarciaSanchez', Ana Salvador-Adriano1, Noe Alberto RamirezGomez', Elizabeth Moreno-Arriola1, Danie/ Ortega-Cuellar ${ }^{2}$ Antonio Velázquez-Arellano ${ }^{1}$

${ }^{1}$ Nutritional Genetics Unit, Instituto de Investigaciones Biomedicas UNAM and Instituto Nacional de Pediatria; ${ }^{2}$ Research Division, Instituto Nacional de Pediatria. Mexico City, Mexico

We found that interfering with ATP production through reduction of oxidative phosphorylation (OXPHOS) by privation of essential cofactors (biotin, thiamine), decreases the mRNAs and proteins for liver glycolysis and lipogenesis, and increases those for fatty acid oxidation and gluconeogenesis. These effects are mediated by the eukaryotic energy sensor AMPK, except in the case of hepatic gluco-
P61

\section{Improvements in Long-Term Weight Loss and Clinical Parameters with the Use of Nutrigenetics in a 2-Year Prospective Study}

\author{
Maria Vranceanu' 1 Lorena Filip ${ }^{2}$, Horatiu Albu³, \\ Ioana Ecaterina Pralea ${ }^{4}$, Marco Perricone ${ }^{5}$, Keith Grimaldi ${ }^{6}$ \\ ${ }^{1}$ Department of Nutrigenetics and Personalized Nutrition, \\ Eurogenetica, Milan, Italy, ${ }^{2}$ Department of Bromatology \\ and Hygiene, luliu Hatieganu University of Medicine and \\ Pharmacy, Cluj Napoca, Romania, ${ }^{3}$ Department of \\ Molecular Nutrition, Columna Medical Center, Bucharest, \\ Romania, Department of Drug Analysis, ${ }^{4}$ Iuliu Hatieganu \\ University of Medicine and Pharmacy, Cluj Napoca, \\ Romania, ${ }^{5}$ Department of Nutrigenetics and Personalized \\ Nutrition, Eurogenetica, Rome, Italy, ${ }^{6}$ Department of \\ Nutrigenetics, Eurogenetica, London, UK
}

Objectives: Genetic variation is known may influence dietary requirements, giving rise to the new field of nutritional genomics and raising the possibility of individualizing nutritional intake for optimal health, disease prevention and better weight management on the basis of an individual's genomeThis study investigated whether the inclusion of genetic information to personalize a patient's diet (nutrigenetics) could improve long term weight management.

Methods: Two groups of patients attending a weight management clinic were prospectively studied. The ketogenic group consisted of 53 patients followed for 24 weeks a ketogenic diet plan with $1600 \mathrm{kcal}$. The nutrigenetics group consisted of 61 patients were offered a nutrigenetic test screening 26 variants in 24 genes involved in metabolism. This group followed a personalized diet with 1600 kcal too and all recommendations based on their DNA. Weight, BMI, total cholesterol, HDLcholesterol and fasting blood sugar levels were monitored.

Results: Both diets group performed well over the 24 weeks but after 2 years the nutrigenetic group fared better on the clinical values of plasma glucose, total cholesterol and HDL. Furthermore after 2 years $75 \%$ of the nutrigenetic patients had maintained weight loss compared to $21 \%$ in the non-genetic group.

Conclusions: Addition of nutrigenetically tailored diets in the weight loss phase and the general healthy eating for life phase resulted in better longer-term BMI reduction and improvements in blood glucose and cholesterol levels 


\section{P62 \\ Association and Gene-Environment Interaction Effect of AGRT1 and VEGFR-2 Gene Polymorphisms with Mental Health on Metabolic Risk Factors of Non- Communicable Diseases in Chinese Malaysian Female Adults}

\author{
Roseline Wai Kuan Yap ${ }^{1}$, Mei-Hua Lin'², Yoshihiro Shidoji3, \\ Wai Sum Yap ${ }^{4}$ \\ ${ }^{1}$ School of Biosciences, Taylor's University, Malaysia; \\ ${ }^{2}$ Faculty of Science \& Technology/Psychology, Sunway \\ University, Malaysia; ${ }^{3}$ School of Health Science, University \\ of Nagasaki, Japan; ${ }^{4}$ Faculty of Applied Sciences, UCSI \\ University, Malaysia
}

Female adults in Malaysia have a higher risk of chronic non-communicable diseases (NCDs) and mental health problems. Associations of rs5186 in angiotensin II type 1 receptor (AGTR1) and rs2071559 of vascular endothelial growth factor receptor-2 (VEGFR-2) with metabolic risk factors of NCDs were reported in Asian populations. Hence, this study examined the association and interaction effects of AGTR1 (rs5186) and VEGFR-2 (rs2071559) gene polymorphisms with mental health on metabolic risk factors of NCDs in Chinese Malaysian female adults. Anthropometric measurements: body mass index (BMI), body fat percentage (BFP), blood pressure; and biomarkers: fasting blood glucose (FBP), glycated hemoglobin (HbA1c), total cholesterol, triglycerides, low-density lipoprotein cholesterol, highdensity lipoprotein cholesterol (HDL-C), CA 15-3 and CA 125 were determined. Job Stress Scale (JSS), Depression, Anxiety, and Stress Scale (DASS-21), and Rhode Island Stress and Coping Inventory (RISCI) questionnaires were used to measure job stress, mental health (stress, anxiety and depression), and coping with perceived stress. Scores from both JSS and DASS-21 are positively correlated with the severity of stress, anxiety, and depression. Higher scores from RISCI indicate better coping with perceived stress. Genotyping of rs5186 and rs2071559 was performed by real-time PCR using Taqman probes. A total of 81 Chinese Malaysian female adults aged 30-65 years old were included in the study. The allele frequencies for rs5186 (A; C allele) and rs2071559 (C; T allele) were 0.96; 0.04 and $0.41 ; 0.59$ respectively. The mean scores obtained from stress, anxiety and depression showed mild or normal levels. The mean score for coping with perceived stress was above average. Significant genetic associations include rs5186 with CA15.3 levels $(\mathrm{p}<0.001)$ and rs2071559 with HbA1c levels $(p=0.034)$ after adjusting for potential confounders. Significant correlations were obtained for stress with BMI $(r=-0.022 ; p=0.046)$, depression with BFP $(r=-0.242 ; p=0.030)$; and stress coping with FBP $(r=0.303 ; p=0.006)$. Significant geneenvironment interaction effects were obtained for rs2071559 with stress $(\mathrm{p}=0.015)$ and $\mathrm{rs} 2071559$ with depression $(\mathrm{p}=0.038)$ on HDL-C levels. Significant associations and/or interaction effects were obtained in a wide range of metabolic risk factors of chronic NCDs. This adds on to the complexity related to the etiology of these diseases, which warrants further investigations.

\section{P63}

MC4R Polymorphism Associated with Antipsychotic-Associated Weight Gain

\author{
Lufei Young ${ }^{1}$, Olivia Dong ${ }^{2,3}$, Tim Wiltshire ${ }^{2,3}$ Kevin Kupzyk $^{4}$, \\ Nicholas Guenze/4 \\ ${ }^{1}$ College of Nursing, Augusta University, Augusta, GA, \\ USA; ${ }^{2}$ Division of Pharmacotherapy and Experimental \\ Therapeutics, UNC Eshelman School of Pharmacy, \\ University of North Carolina at Chapel Hill, Chapel Hill, NC, \\ USA; ${ }^{3}$ Center for Pharmacogenomics and Individualized \\ Therapy, University of North Carolina at Chapel Hill, \\ Chapel Hill, NC, USA; ${ }^{4}$ College of Nursing, University of \\ Nebraska Medical Center, Omaha, NE, USA
}

Background: Atypical antipsychotic medications have been linked to weight gain and other metabolic complications. Some of the variance in antipsychotic metabolic side effects may be related to genetic factors.

Objectives: Identify genetic risk markers that are predictive of antipsychotic-associated weight gain (AAWG) for patients prescribed atypical antipsychotic drugs.

Methods: A secondary analysis was completed using medical records from 447 patients at outpatient psychiatric clinics in Southeast Nebraska from 2009 to 2016. The IRB at the University Nebraska Medical Center and the clinic's Ethics Committee approved of this study. The TaqMan single nucleotide polymorphism (SNP) genotyping assay (Life Technologies, Grand Island, New York) was used for genotyping. Medication and genetic information were required to be included in the analysis. An analysis of covariance (ANCOVA) was used to compare BMI mean values, and clinical variables were included as covariates. A Bonferroni correction was applied to adjust for multiple comparisons. The clinical variables included in the model as covariates were: age, gender, medications, diagnoses, and number of chronic conditions. The tested genetic variants included 14 SNPs in 13 candidate genes associated with obesity: SLC6A4, 5-HTTLPR, CACNA1C, ANK3, 5HT2C, MC4R, DRD2, COMT, $A D R A 2 A, M T H F R, B C N F$, OPRM1, and GRIK1.

Results: The analysis included 440 subjects ( $43 \%$ male, mean age: $35 \pm 19$ ). Approximately $80 \%$ of patients had a diagnosis of refractory anxiety disorder followed by major depressive disorder (62\%), ADHD (35\%), bipolar disorder (17\%), PTSD (11\%), and schizophrenia (3\%). Based on the BMI categories, 152 patients were obese $(36 \%), 129$ were overweight $(31 \%), 136$ were normal $(31 \%)$ and 4 were underweight (1\%). After adjusting for clinical factors, the AA genotype at rs6265 in the $M C 4 R$ gene was significantly associated with a $17.61 \%$ increase in $\mathrm{BMI}(\mathrm{F}[2,440]=6.027, \mathrm{P}=0.003)$.

Conclusion: The analysis suggests the rs 6265 polymorphism in $M C 4 R$ might be a predictive maker of AAWG. Using predictive genetic markers may help identify those at risk of weight gain to provide targeted nutrition, exercise, and pharmacological interventions. Prospective studies are needed to validate these findings. 


\section{P64 \\ Low dosage of Chito-Oligosaccharide (COS) Supplementation Increases the Population of Prevotella in the Cecal Contents of Weanling Pigs}

Ting Y $u^{2}$, Yu Wang ${ }^{1}$, Shicheng Chen', Zhiling Wang', Cui Zhu' ${ }^{2}$, Zongyong Jiang ${ }^{1}$, Xianyong Ma', Guozhong Wu ${ }^{4}$, Zhuang Chen ${ }^{2}$, Chuntian Zheng ${ }^{1}$

${ }^{1}$ Ministry of Agriculture Key Laboratory of Animal Nutrition and Feed Science (South China), Guangdong Public Laboratory of Animal Breeding and Nutrition, Institute of Animal Science, Guangdong Academy of Agricultural Sciences, Guangzhou, China; ${ }^{2}$ Agro-biological Gene Research Center, Guangdong Academy of Agricultural Sciences, Guangzhou, China; ${ }^{3}$ Department of Microbiology and Molecular Genetics, Michigan State University, East Lansing, USA; ${ }^{4}$ Shanghai institute of applied physics, Chinese Academy of Sciences. Shanghai, China.

Introduction: Chito-oligosaccharide (COS) addition promoted animal growth, increased feed digestibility, and reduced the incidence of diarrhea in weaned piglets. To investigate its influence of COS supplementation on piglets gut microbiota and compare the differences of community composition between $\mathrm{COS}$ and antibiotics with $\mathrm{ZnO}$ addition, we assessed the cecal microbial community by $16 \mathrm{~S}$ rRNA gene sequencing with three treatments consisting of basal diet (CTR group), basal diet with COS (COS group), and basal diet with antibiotic and $\mathrm{ZnO}$ (AZ group).

Methods: A total of 60 male weaned piglets (Duroc $\times$ Landrace $\times$ Large White) with an average weight of $6 \mathrm{~kg}$ and 21-day old were used in this study. The control (CTR) group was the piglets fed with basal diet; the antibiotics/ZnO (AZ) treatment group was those given the basal diet supplemented with aureomycin $(30 \mathrm{mg} / \mathrm{kg})$, polymyxin $\mathrm{E}(12 \mathrm{mg} / \mathrm{kg})$ and $\mathrm{ZnO}(3000 \mathrm{mg} / \mathrm{kg})$; the COS group was those given the basal diet supplemented with low-molecular weight $(20,000 \sim 30,000 \mathrm{Da})$ of chitosan $(50 \mathrm{mg} / \mathrm{kg})$. Out of the 60 animals, each treatment consisted of five replicates and 4 piglets per replicate (totally 20 animals). The feeding trials lasted for 28 days and the contents of cecum were collected.

Results: Beta diversity analysis showed that community structure were distinctly difference among above three treatments. Compared with CTR, samples of COS and AZ enriched bacteria affiliated with Prevotella but decreased Firmicutes. Moreover, COS increased the relative abundance of Succinivibrio and Anaerovibrio when compared with AZ. Through microbial functions prediction, COS had higher abundance of functions involved in metabolism of cofactors and vitamins when compared with CTR and AZ. Cecal pH in COS was lower than that in CTR or AZ.

Conclusions: COS could be a natural safe candidate feed additive as an alternative of the feed-grade antibiotics in early-weaned piglets.

\section{P65}

Genetic Variants in Key Enzymes of Choline Metabolism and Maternal Choline Intake during Pregnancy Influence the Risk of Preterm Delivery and Choline Metabolism in Preterms Receiving Total Parenteral Nutrition

\author{
Jie Zhu ${ }^{1,2}$, Bei Wang ${ }^{3}$, Wei Cai ${ }^{1,2}$, Martin Kohlmeier ${ }^{4}$ \\ ${ }^{1}$ Department of Nutrition, Shanghai Jiao Tong University \\ School of Medicine; ${ }^{2}$ Shanghai Key Laboratory of Pediatric \\ Gastroenterology and Nutrition; ${ }^{3}$ Department of \\ Obstetrics, Xinhua Hospital Affiliated to Shanghai Jiao \\ Tong University School of Medicine; ${ }^{4}$ Department of \\ Nutrition \& Nutrition Research Institute, University of \\ North Carolina at Chapel Hill, School of Medicine and \\ Public Health, NC, USA
}

Background/Aims: Adequate choline intake is important for optimal pregnant outcome and neonatal development, which is influenced by single nucleotide polymorphisms (SNPs) of its key enzymes. We sought to investigate the effects of SNPs in phosphatidylethanolamine N-methyltransferase (PEMT) and betaine-homocysteine S-methyltransferase (BHMT) genes, and choline intake during pregnancy on the risk of preterm delivery, choline metabolism and growth in preterms receiving total parenteral nutrition (TPN).

Methods: 100-paired Chinese healthy parturients with term infants and 100-paired parturients with preterm delivery and their preterms were recruited. Dietary choline intake during pregnancy was determined by food frequency questionnaires. Plasma choline levels in peripheral vein from all parturients and preterms were measured with PEMT (-744 $\mathrm{G} \rightarrow \mathrm{C}$, rs12325817) and BHMT (+742 $\mathrm{G} \rightarrow \mathrm{A}$, rs3733890) genotyped.

Results: Plasma choline level in maternal peripheral vein was associated with dietary choline intake during pregnancy $\left(\mathrm{r}^{2}=0.78, P\right.$ $<0.05$ ). Maternal plasma choline levels was related to choline levels in preterm umbilical artery $\left(\mathrm{r}^{2}=0.80, P<0.05\right)$. Among the preterm parturients whose choline intake within the lowest quartile level during pregnancy, PEMT $-774 \mathrm{C}>\mathrm{G}$ increased the preterm risk (OR $2.18,95 \%$ CI $1.29-3.56, P<0.05)$. Plasma choline levels in peripheral vein of preterms were decreased after 7-day TPN feeding; Compared with PEMT-744GG and GC genotypes, the maternal variant $\mathrm{CC}$ genotype resulted in lower plasma choline concentrations in preterms after 7-day TPN feeding $(P<0.05)$. Additionally, the BHMT +742AA genotype further reduced plasma choline levels in preterms after 7-day TPN support $(P<0.05)$, compared with BHMT +742GG and GA genotypes. Moreover, BHMT $+742 \mathrm{~A}$ allele increased the difficulty in regaining birth weight after 11-day TPN feeding (OR 1.65, 95\% CI, 1.19-2.78, $P<0.05$ ), compared to the G allele.

Conclusions: Maternal PEMT-744CC genotype and inadequate choline intake during pregnancy may increase the risk of premature births. Maternal PEMT-744CC genotype and neonatal BHMT +742AA genotype may further increase susceptibility of choline decrement in preterms on TPN feeding. BHMT $+742 \mathrm{~A}$ allele may inhibit the weight gain of preterms who may need more choline for growth and development after birth. 


\section{P66}

\section{Early-Life Exposure to Permethrin Food Contaminant Inducing Neurodegeneration: Is It Matter of Epigenetics?}

Laura Bordoni ${ }^{1}$, Cinzia Nasuti ${ }^{2}$, Donatella Fedeli², Rosita Gabbianelli²

'School of Advanced Studied and ${ }^{2}$ School of Pharmacy, University of Camerino, Italy

Permethrin (PERM) exposure in rats at a dose close to No Observed Adverse Effect Level (NOAEL) for 15 days during neonatal brain development leads to the accumulation of the pesticide long after exposure and to the impairment of dopaminergic system in striatum nucleus, contributing later in life to the burden of oxidative stress and behavioral changes linked to a Parkinson's like syndrome through several pathways. Among these, epigenetics is a strong candidate mechanism to be involved because of its well-known capability to modulate the effect that environmental factors have on the genome. Previous studies showed alteration of DNMT's expression regulation and global DNA methylation both in the exposed and in F1 generation. Anyway the dynamic of the epigenetic contribution to the pathology onset is still not clear. In order to clarify the role of epigenetics in this Parkinson-like syndrome induced by a food contaminant, rats were gavaged through intragastric tube with PERM solubilized in corn oil at a dose of 1/50 of LD50 corresponding to $34.05 \mathrm{mg} / \mathrm{kg}$. The compound was administered daily in the morning from PND6 to PND21 and control group was administered with vehicle. Animals where than sacrificed at 60PND and the expression of genes involved in the dopaminergic pathway, also related in Parkinson onset (Nurr1, TH, $\alpha$-syn), and genes that regulate epigenetic mechanisms specifically in the brain (Mecp2, TET1, TET2, TET3) was analyzed. Global DNA methylation level assessed by ELISA and histone modifications at specific gene loci investigated through ChIP were also determined in order to clarify the epigenetic landscape. Significant alteration in terms of these markers has been identified in our model, suggesting that epigenetics can exert a pivotal role in the onset of this disease. This study focus on an emerging role of food intake, specifically in early-life, in the regulation of the onset later in life of chronic diseases through intriguing mechanisms such as epigenetics. 
Abbott, K. P8

Adamski, M. P5

Aguilar-Salinas, C.A. P41

Albu, H. P61

Albuquerque, R.C. P12

Alderete, T.L. P30

Alkayal, F. P6

Amos, A. P7

Araújo, J.N.G. P15

Arcidiacono, T. P57

Arefanian, H. P6

Arguello, L.E. P37

Arias, L. P22

Bailey, S.M. P29

Barreto, A.L. P20

Barrington, W. O1

Batinga, L.B. P17

Beckett, E.L. P8

Bennett, B. O1

Ben-Nissan, D. P9

Bharatraj, D.K. P58

Bjellaas, T. P39

Björkegren, J.L.M. P52

Bonaldi, A. P20

Bordoni, L. P10, P66

Bourges-Rodriguez, H. P59

Bramucci, M. P10

Brasacchio, C. P57

Brassard, D. P46

Bui, H.H. P39

Cai, W. P65

Campos-Pérez, F. P41

Campos-Pérez, W. P11

Canizales-Quinteros, S. P41

Cantor, R.M. P52

Cárcamo, J.G. P22

Cavadino, A. O3

Chan, B. P35

Charugundla, S. P21, P33

Che, N. P21, P33, P52

Chen, S. P64

Chen, Z. P64

Choi, W.-S. P31

Chowanadisai, W. P25

Civelek, M. P52

Collins, J. P5

Cominetti, C. P26

Costa, G.R. P12

Couture, P. P46

Cruz, M.S.M. P15

Cuervo, M. P48

Cunha, A.T.O. P15
Daafar, M. P43

Diaz, R.S. P20

Dong, O. P13, P63

Drevon, C.A. P39, P52

Duarte, M.K.R.N. P15

Duarte, V.H.R. P15

Duerksen-Hughes, P. P16, P18

Ecaterina Pralea, I. P61

Eck, B.L.P. P46

Eroshkin, A. P16, P18

Eskin, E. P21

Fedeli, D. P10, P66

Ferguson, J. P8

Fernandes, C.T.O. P15

Ferreira, N.I. P17

Filip, L. P61

Filippov, V. P16, P18

Filippova, M. P16, P18

Fiorini, D. P10

Fisberg, R.M. P19

Fonseca, A.C. P20

Fraser, G.E. P16, P18

Freitas, R.C.C. P15

Fujii, T.M.M. P19, P20

Fuqua, B. P21

Gabbianelli, R. P10, P66

Gallardo, M.A. P22

Garcia-Lacarte, M. P23

Garcia-Sanchez, J.A. P60

Garg, M. P8

Geary, B.A. P30

Geoffroy, C. P22

Gharakhanian, R. P52

Gigleux, I. P46

Goni, L. P48

Gonzalez-Aldaco, K. P53

González-Muniesa, P. P36

Goran, M.I. P30

Goyal, D. P37

Grimaldi, K. P61

Guenzel, N. P63

Gumilar, E. P43

Gundersen, T.E. P39

Gupta, P. P24

Gupta, S. P39

Hart, M.D. P25

Hasin-Brumshtein, Y. P39, P52

Hassimotto, N.M.A. P12

Hazen, S. P41

Hazen, S.L. P34, P39
He, C. P28

Heber, D. O4, P35

Henning, S.M. O4, P35

Hernandez, S. P22

Hernandez-Pando, R. P41

Hernandez-Vazquez, A.J. P60

Hevener, A.L. P39

Horst, M.A. P20, P26

Horvath, S. P44

Howe, S. O1

Hsu, M. O4, P35

Huang, L. P27

Huang, Y. P28

Huertas-Vazquez, A. P41

Hui, S. P21, P33, P41

Hui, S.T. P29, P39

Hwang, D.H. P30

Hyppönen, E. O3

Ibarra-González, I. P41

Jaceldo-Siegl, K. P16, P18

Jerome, K.N. P25

Jiang, Z. P64

Jin, Z. O4

Jones, P.J.H. P46

Jones, R.B. P30

Karani, V. O3

Kim, E.D. P52

Kim, H.-N. P31

King, K. P8

Kohlmeier, M. P13, P65

Koistinen, H.A. P52

Koplev, S. P52

Krauss, N.B.R.M. P34

Krishnan, K.C. P33, P39, P52

Kupzyk, T.W.K. P63

Kurtz, I. P34

Laakso, M. P52

Lajolo, F.M. P12

Lang, J.M. P34

Lee, J. P39

Lee, R. O4

León-Mimila, P. P41

Levine, M.E. P44

Levy, E. P46

Li, D.Y. P39

Li, Q. P35, O4

Li, Y. O2

Li, Z. P35, O4

Lima, S.C.V.C. P15
Lin, M.-H. P62

Long, J. P35, O4

Lopes, C.R.M. P17

López-Contreras, B. P41

López-Pascual, A. P36

Lorente-Cebrián, $\mathrm{S}$.

Loskutov, V. P16, P18

Lovegrove, J.A. O3, P26

Lu, D. P21

Luca, M.D. P29

Luchessi, A.D. P15

Lucock, M. P8

Lusis, A.J. P21, P29, P33, P34, P39, P41, P52

Ma, X. P64

Macías-Kauffer, L. P41

Macrina, L. P57

Mäkinen, S. P52

Mansego, M.L. P23

Marafie, S. P6

Marchioni, D.M.L. P19

Maria-Engler, S.S. P12

Martin, A.A. P30

Martin, C. P8

Martinez, J.A. O5, P23

Martínez, J.A. P36, P48, P50

Martínez-López, E. P11

Mauldin, K. P37

Mehrabian, M. P39, P52

Méndez-Sanchez, N. P41

Mendoza-Fandino, G.A. P38, P45

Meng, Y. P52

Mezzavilla, M. P57

Michels, K.B. P55

Milagro, F.I. O5, P23, P48, P50

Monggesang, F. P43

Monoarfa, Y. P43

Morais, C.C. P26

Moreno-Arriola, E. P60

Mosier, M. P39

Murgia, C. P5

Nagalla, B. P58

Nasuti, C. P10, P66

Niblett, S. P8

Norde, M.M. P19

Norheim, F. P21, P33, P39, P52

Novak, R. P39

Ocampo-Medina, E. P41

Ojeda-Granados, C. P42, P49, P53 
Oliveira, J.M. P15

Oliveira, V.R. P17

Ong, T.P. P12

Ortega-Cuellar, D. P60

Otoluwa, A.S. P43

Palmer, S.L. P30

Pan, C. P21, P29, P33, P39, P52

Panduro, A. P42, P49, P53

Parks, B. P21

Parks, B.W. P39, P52

Pedrosa, T.N. P12

Pérez-Robles, M. P11

Perricone, M. P61

Phadke, A. P24

Pimentel, J.B. P15

Pointer, M. P13

Pomp, D. O1

Qi, H. P33

Qin, H. P27

Quach, A. P44

Quassinti, L. P10

Quintero, M.D. P38, P45

Rajbhandari, P. P52

Rajendiran, E. P46

Ramírez, C. P47

Ramirez-Gomez, N.A. P60

Ramos-López, O. O5, P48

Ramprasath, V. P46

Randolph, J.M. P54

Reue, K. P52

Reyes-Fermín, L. P41

Riezu-Boj, J.I. O5, P48
Rivera-Iñiguez, I. P42, P49, P53

Rogero, M.M. P19

Rojas, C.M. O1

Roman, S. P42, P49, P53

Rosenberg, G.M. P52

Sabir, S. P33

Sailoo, A.K.K. P58

Salvador-Adriano, A. P60

Samblas, M. P50

Santos, D.M.C. P15

Sawrey-Kubicek, L.R. P54

Schellenberger, M. P51

Seldin, M.M. P52

Sepulveda-Villegas, M. P49, P53

Sepúlveda-Villegas, M. P42

Shawaf, E.A. P6

She, Y. P46

Shen, T. P27

Shidoji, Y. P62

Shih, D.M. P52

Silbiger, V.N. P15

Silcock, L. P57

Silva, A.M.G. P15

Smith, B.J. P25

Soldati, L. P57

Song, S.-W. P31

Souza, A.L.S. P15

Souza, T.O. P15

Steinberg, F.M. P54

Stevens, A.M. P54

Stiemsma, L.T. P55

Strong, M.D. P25

Sun, C. O2
Surendran, S. P26

Suzuki, O. P13

Swain, T.M. P29

Tabaie, W.E. P56

Tang, T.Z. P25

Tao, T. P28

Terranegra, A. P57

Tessier-Grenier, M. P46

Thaha, A.R. P43

Threadgill, D. O1

Tiss, A. P6

Tomei, S. P57

Tontonoz, P. P52

Torres-Castillo, L. P11

Torrinhas, R.S.M.M. P15

Tremblay, A. P46

Twohig, C. P5

Varakantham, V. P58

Vasquez-Trespalacios, E.M. P38

Vega-Badillo, J. P41

Vela-Amieva, M. P41

Velásquez, L. P45

Velazquez-Arellano, A. P59, P60

Vergnes, L. P52

Veysey, M. P8

Vezzoli, G. P57

Vilhena, C. P20

Vilhena, M. P20

Villamil-Ramírez, H. P41

Vimal, K.S. P26

Vranceanu, M. P61

Vulpe, C. P21
Wang, B. P65

Wang, C. P16, P18

Wang, K.G. P25

Wang, Y. P64

Wang, Z. P64

Wilson Tang, W.H. P39

Wiltshire, T. P13

Wirdjatmanto, B. P43

Woo, S.L. P35

Wu, G. P64

$\mathrm{Wu}, \mathrm{X} . \mathrm{O} 2$

Yamada, M. P20

Yan, X. P27

Yang, H. P28

Yang, J. P35, O4

Yang, X. P28

Yap, R.W.K. P62

Yap, W.S. P62

Yatim, H. P43

Yin, Y. P28

Young, L. P63

$\mathrm{Yu}, \mathrm{T}$. P64

Zheng, C. P64

Zhou, A. O3

Zhou, S. P28

Zhou, Z. P33

Zhu, C. P54, P64

Zhu, J. P65

Zivkovic, A.M. P54

Zulet, M.A. P23, P50 